\author{
Banco de México \\ Documentos de Investigación
}

$\mathrm{N}^{\circ} 2021-12$

\title{
Inversión Extranjera Directa y Productividad Laboral en la Industria Manufacturera Regional
}

\author{
Erick Rangel González \\ Banco de México
}

\author{
Luis Fernando López Ornelas \\ Universidad de Guanajuato
}

\section{Agosto 2021}

La serie de Documentos de Investigación del Banco de México divulga resultados preliminares de trabajos de investigación económica realizados en el Banco de México con la finalidad de propiciar el intercambio y debate de ideas. El contenido de los Documentos de Investigación, así como las conclusiones que de ellos se derivan, son responsabilidad exclusiva de los autores y no reflejan necesariamente las del Banco de México.

The Working Papers series of Banco de México disseminates preliminary results of economic research conducted at Banco de México in order to promote the exchange and debate of ideas. The views and conclusions presented in the Working Papers are exclusively the responsibility of the authors and do not necessarily reflect those of Banco de México. 
Documento de Investigación 2021-12
Working Paper

2021-12

\title{
Inversión Extranjera Directa y Productividad Laboral en la Industria Manufacturera Regional*
}

\author{
Erick Rangel González ${ }^{\dagger}$ \\ Banco de México \\ Luis Fernando López Ornelas \\ Universidad de Guanajuato
}

\begin{abstract}
Resumen: La Inversión Extranjera Directa (IED) es a menudo señalada como un factor impulsor del crecimiento económico, si bien no existe un consenso en la evidencia empírica a nivel internacional respecto a su efecto sobre la productividad laboral. El presente documento analiza los efectos de la Inversión Extranjera Directa sobre la productividad laboral en el sector manufacturero en México durante el periodo 2007-2015 utilizando datos panel y empleando como unidad de análisis a las entidades federativas del país. Las estimaciones se realizan empleando el método generalizado de momentos, el cual permite considerar posibles problemas de endogeneidad. Los resultados indican un efecto positivo y estadísticamente significativo de la IED como proporción del PIB manufacturero sobre la tasa de crecimiento de la productividad laboral estimada a partir del Índice de Productividad Laboral de las Manufacturas publicado por INEGI. Resultados similares se encuentran si el crecimiento en la productividad laboral se estima por medio del PIB manufacturero por trabajador, si bien estos últimos cuentan con menor poder estadístico en algunas especificaciones.
\end{abstract}

Palabras Clave: Productividad Laboral, Inversión Extranjera Directa, Manufacturas, México.

JEL Classification: J01, J24, Q29, R11

Abstract: Foreign Direct Investment (FDI) is often identified as a driver of economic growth, although there is no consensus on this topic in the international empirical evidence regarding its effect on labor productivity. This document analyzes the effects of Foreign Direct Investment on labor productivity in the manufacturing sector in Mexico during the 2007-2015 period by using panel data and federative entities as unit of analysis. The estimates are calculated by the generalized method of moments, which allows to consider for possible endogeneity problems. The results indicate a positive and statistically significant effect of FDI as a proportion of manufacturing GDP on the growth rate of labor productivity when the latter is estimated with the Manufacturing Labor Productivity Index published by INEGI. Similar results are found if growth in labor productivity is estimated by using manufacturing GDP per worker, although the latter have less statistical power in some specifications.

Keywords: Labor Productivity, Foreign Direct Investment, Manufacturing, Mexico.

\footnotetext{
*Agradecemos los comentarios de Alejandrina Salcedo, el editor asociado, los dictaminadores anónimos del Banco de México, y el apoyo de Sara Hutchinson.

† Dirección General de Investigación Económica. Correo: erick.rangel@banxico.org.mx.

$\ddagger$ Universidad de Guanajuato. Correo: flopezornelas@gmail.com.
} 


\section{Introducción}

La productividad laboral, en el largo plazo, es uno de los factores más importantes que inciden en el nivel de vida de la población, ya que afecta el poder adquisitivo de los hogares a través de mayores salarios y/o menores precios (Harris, 2002). Adicionalmente, reduce los costos de producción y aumenta la rentabilidad de las inversiones en las empresas (OIT, 2008). En efecto, en el modelo neoclásico microeconómico la relación entre la productividad laboral y los salarios es directa ya que las empresas maximizadoras de beneficios contratan dicho factor hasta el punto en que su producto marginal iguala su costo (salarios). Desde el punto de vista macroeconómico, de acuerdo a Solow (1957), la productividad laboral es el determinante que tiene mayor influencia sobre el nivel de ingresos de un país.

El objetivo de este estudio es analizar el efecto de la Inversión Extranjera Directa (IED) sobre la productividad laboral en el sector manufacturero mexicano durante el periodo 2007-2015 utilizando datos panel y empleando como unidad de análisis a las entidades federativas del país. En específico, se explota la heterogeneidad en la productividad laboral promedio de los estados del país en la industria manufacturera y de otras variables, para identificar el efecto de la IED sobre dicha productividad, considerando posibles problemas de endogeneidad y/o de variable omitida. Las estimaciones principales son realizadas a través del método generalizado de momentos empleando la técnica propuesta por Blundell y Bond (1998). Además, la especificación empírica permite controlar por diversos factores que influyen sobre el crecimiento económico como la infraestructura, cambios en el factor trabajo, la apertura comercial, el desarrollo de los mercados financieros y la inflación.

Las estimaciones econométricas principales se realizan utilizando la tasa de crecimiento del Índice de Productividad Laboral de las Manufacturas (IPLM) calculado y publicado por el INEGI. Dicho índice es una medida construida a partir de la producción total, a diferencia de las medidas tradicionales que emplean el valor agregado. Adicionalmente, para dimensionar los niveles de productividad por entidad federativa se incluye un análisis descriptivo del Producto Medio por Trabajador (PMT) estimado a través de la Encuesta Mensual de la Industria Manufacturera (EMIM), el cual también se construye a partir de la producción total. Asimismo, se presentan estimaciones utilizando el PMT y el PIB a precios constantes de 
2013 por trabajador en la industria manufacturera (que se basan en el concepto de valor agregado). Las estimaciones sugieren que, una vez que se controla por posibles problemas de endogeneidad y/o variable omitida, y por otros elementos que influyen en el crecimiento económico, la IED como proporción del PIB tiene un impacto positivo y estadísticamente significativo sobre la tasa de crecimiento del IPLM y dichos resultados se mantienen empleando diferentes especificaciones econométricas y métodos de estimación. Asimismo, se obtienen resultados similares cuando se emplea el PMT como medida de productividad laboral. Por su parte, si se mide la productividad laboral por medio del PIB manufacturero por trabajador (PIBMT), la significancia estadística de la IED es más sensible a la especificación econométrica y método de estimación utilizado, si bien se observa un coeficiente positivo en todas las estimaciones, y las especificaciones no significativas se encuentran, en su mayoría, no muy alejados de los límites estadísticos de significancia.

La mayor parte de los estudios sobre productividad y productividad laboral están basados en estimaciones que emplean medidas de valor agregado. Sin embargo, existe un creciente interés en estimar funciones de producción y analizar la productividad a través de la producción total (Amiti y Konings, 2007; Basu y Fernald, 1995; Gandhi, Navarro y Rivers, 2020; Kasahara y Rodrigue, 2008; Levinsohn y Petrin, 2003). ${ }^{1}$ La diferencia entre ambos conceptos de productividad es más pronunciada a nivel sectorial o industrial que a nivel agregado (Cobbold, 2003). Al excluir los insumos intermedios, las estimaciones del crecimiento de la productividad basadas en el valor agregado niegan una fuente importante de crecimiento económico (Gullickson,1995). En el sector manufacturero los insumos intermedios constituyen una parte considerable de la estructura de costos. Así, una especificación de productividad que excluye los insumos intermedios hace que sea más

\footnotetext{
${ }^{1}$ El uso del valor agregado en las funciones de producción implica algunos supuestos restrictivos: i) en el mundo real las empresas no producen cosas en unidades de valor agregado sino en unidades de producto (Oulton y O'Mahony, 1994); ii) El modelo de valor agregado supone que la función de producción es aditiva y separable (Jorgenson et al., 1987); iii) asumen que los precios de los productos finales y los insumos intermedios se incrementan en la misma proporción (Jorgenson et al., 1987); iv) la producción bruta es la medida natural de la producción de una empresa o industria, mientras que el valor agregado es un índice económico sin interpretación física. Por lo tanto, el uso del valor agregado asume implícitamente competencia y rendimientos constantes a escala (Basu y Fernald, 1995).
} 
probable la medición incorrecta de las tendencias de crecimiento y limita los tipos de análisis que se pueden realizar con este tipo de medidas de productividad (Gullickson, 1995). ${ }^{2}$

En el caso específico de la productividad laboral, esta también puede ser medida por medio del valor agregado y de la producción agregada. Por un lado, las medidas de productividad laboral basadas en valor agregado tienen la ventaja sobre su contraparte (producción agregada) de ser menos sensibles a la sustitución entre el factor trabajo y los insumos intermedios (Cobbold, 2003). Por otro lado, tienen la desventaja de ser sensibles al tratamiento de los precios y la contabilidad del factor trabajo. En específico, la variable de productividad laboral estimada en base al producto total empleada en este estudio (IPLM) conlleva un proceso muy cuidadoso de tratamiento en los precios de los productos, mientras que las variables de valor agregado estimadas en base al PIB por trabajador son sensibles al índice de precios que se usa para deflactar. Cabe señalar que los temas relacionados con los precios no son menores, sobre todo si se considera que el crecimiento anual de los precios es mayor al de la productividad laboral en el periodo analizado. Otra ventaja que tiene el IPLM sobre el PIBMT es que realiza una contabilidad más precisa del factor trabajo. ${ }^{3}$

Si bien se ha argumentado que la IED podría estimular la productividad laboral por medio de cadenas de producción mejor integradas a los procesos productivos globales, imitación de procesos productivos, creación de nuevos productos y procesos, y capacitación en el empleo, la evidencia empírica no refleja un consenso a favor de dicho argumento. Así, el presente documento de investigación pretende contribuir al entendimiento de los efectos de la IED sobre la productividad laboral, y en específico sobre su tasa de crecimiento. Dicho tema es de gran importancia pues la productividad laboral influye directamente sobre el nivel de vida de la población. Asimismo, el estudio desde el punto de vista regional de los flujos de inversión extranjera directa es relevante pues aporta información sobre la pertinencia de

\footnotetext{
${ }^{2}$ Jorgenson et al. (1987) muestran que los insumos intermedios son la fuente predominante de crecimiento de la producción a nivel industrial en Estados Unidos, superando tanto el crecimiento de la productividad como las contribuciones de capital y trabajo en la gran mayoría de las industrias.

${ }^{3}$ En la sección de resultados se amplía la discusión sobre las diferencias entre el IPLM y el PIB por trabajador del sector manufacturero.
} 
diseñar políticas públicas de atracción de IED a nivel regional que ayuden a incrementar la productividad laboral y con ello el nivel de vida de sus habitantes.

El documento de investigación está organizado de la siguiente forma: la sección dos muestra una revisión de la literatura; la sección tres contiene un análisis de la evolución de la productividad laboral y de los niveles de esta por entidad federativa; la descripción de los datos y del modelo econométrico se presentan en las secciones cuatro y cinco; finalmente, la sección seis contiene las conclusiones y consideraciones finales de la presente investigación.

\section{Revisión de la Literatura}

Existen diversas teorías que relacionan los flujos de IED con la productividad laboral y el crecimiento económico. El modelo de variedad de productos de Romer (1990) argumenta que el crecimiento en productividad es derivado de una expansión en la variedad de productos intermedios especializados. Así, la IED podría permitir incrementos en la productividad si incrementa la variedad de productos intermedios en la economía. Por su parte, Mitze y Özyurt (2014) mencionan que la IED es una fuente de empleo y acumulación de capital físico, lo que a su vez aumenta la productividad laboral. Asimismo, la IED podría estimular la productividad en economías en desarrollo, ya que genera la llegada de empresas

trasnacionales las cuales pueden contar con sistemas de producción más eficientes e integrados a las cadenas de valor global en relación a las empresas domésticas.

Zhao y Zhang (2010) señalan que, por un lado, la IED puede promover la productividad a través de cadenas de producción, imitación de procesos productivos, creación de nuevos productos y procesos, y capacitación en el empleo. No obstante, también comentan que mucha de la inversión es hecha por compañías transnacionales que algunas veces restringen la difusión de nuevas tecnologías y conocimiento especializado en sus subsidiarias, y en tal caso no habría un impacto sobre la productividad laboral. Más aún, se menciona que la IED podría generar un impacto negativo sobre la productividad al inhibir el desarrollo tecnológico en los países receptores si las compañías multinacionales desean limitar la innovación y uso de la tecnología en los países receptores de IED (Ram y Zhang, 2002). Asimismo, Fillat y Woerz (2011) mencionan que la IED puede generar un efecto de desplazamiento sobre la 
inversión doméstica privada generando una menor productividad y crecimiento económico. Por su parte, Alfaro et al. (2004) argumentan que la falta de un sistema financiero bien desarrollado puede limitar los impactos positivos de la IED sobre el crecimiento económico.

En la literatura se pueden identificar diversas formas de analizar la productividad laboral: algunos estudios lo hacen empleando el producto medio por trabajador y su tasa de crecimiento (Belorgey et al., 2006; Bourlès y Cette, 2007; Choudhry et al., 2016); mientras que otros utilizan la tasa de crecimiento del producto menos la tasa de crecimiento del factor trabajo como unidad de análisis (Gust y Marquez, 2004); un tercer enfoque consiste en emplear los salarios promedio como medida de la productividad laboral y a través de estos analizar algunos de los determinantes más importantes (Carlino y Voith, 1992; Decker et al., 2009).

La literatura empírica muestra evidencia mixta sobre los efectos de la IED sobre la productividad laboral. Entre los estudios que encuentran una relación positiva se encuentra el trabajo de Mitze y Özyurt (2014) para las provincias en China. Sus resultados indican que en el largo plazo la productividad laboral regional es afectada por los efectos espaciales directos e indirectos de la IED y el comercio internacional, además de otros factores como la infraestructura regional y la dotación de capital físico. Similarmente, Zhao y Zhang (2010) encuentran que la IED afecta tanto de manera directa, como a través de efectos de derrama, a la productividad industrial en China tanto en niveles como en su tasa de crecimiento. Hejazi y Safarian (1999) encontraron evidencia de que la IED es un canal efectivo para la difusión de investigación y desarrollo para los países de la OCDE y que su relevancia es aún mayor que la del comercio de bienes y servicios. Por su parte, Yazdan y Hossein (2013) analizan los impactos de la IED y de la inversión en tecnologías de comunicación para países de medio oriente durante el periodo 1990-2010, y encuentran efectos positivos de ambas variables sobre la productividad.

En referencia a estudios menos concluyentes sobre los efectos de la IED, Carkovic y Levine (2005) emplean técnicas de panel dinámico para 72 países y no encuentran evidencia robusta de los efectos de la IED sobre el crecimiento económico. Similarmente, Borensztein et al. (1998) emplean datos de panel para 69 países para las décadas de 1970 y 1980 encontrando 
una relación positiva de la IED sobre la productividad laboral solamente para los países que rebasaban un nivel mínimo de capital humano. Por su parte, Mei y Been-Lon (2000) encuentran para el sector manufacturero en Taiwán, que incrementos en el tamaño del sector exportador y en la IED aumentan la productividad laboral en empresas de tamaño pequeño, pero la reducen en empresas grandes. Dimelis y Papaioannou (2010) empleando un panel para 43 países en el periodo 1993-2001 encuentran una relación positiva entre la IED y la productividad laboral. Sin embargo, cuando dividen la muestra entre países desarrollados y en vías de desarrollo no encuentran evidencia a favor de tal relación para los últimos. Similarmente, Fillat y Woerz (2011) usando una muestra de 35 países (avanzados y en niveles de desarrollo medio) durante el periodo 1987-2002 encuentran que los efectos de la IED sobre la productividad dependen del grado de desarrollo de las economías, si bien en su caso los efectos más modestos se observan en las economías más avanzadas. Analizando por sectores, Alfaro (2003) encuentra un efecto negativo de la IED sobre el sector primario, positivo sobre el sector manufacturero y ambiguo sobre el sector servicios y la economía en general.

En el caso específico de la economía mexicana, Olechko (2004) estudia la relación existente entre la productividad laboral de un grupo de empresas manufactureras de México y la inversión extranjera directa en la década de los noventa, encontrando evidencia de que la presencia de inversión extranjera en el sistema económico mexicano tiene un impacto sobre la productividad; sin embargo, este efecto no se observa para las empresas domésticas, lo que sugiere una falta de mecanismos para que los negocios locales captaran los efectos de derrama de la inversión extranjera. Por su parte, Casanueva y Rodríguez (2009) encuentran evidencia que indica que la IED tiene impactos positivos sobre la productividad laboral en la industria manufacturera mexicana, y que sus efectos se incrementan en la medida en que existe un ambiente de trabajo de calidad. Similarmente, Mendoza y Cabrera (2014) plantean que los flujos de inversión extranjera directa tienen un impacto positivo sobre la productividad laboral en la frontera norte de México. Cabe señalar que ninguno de los estudios mencionados emplea datos de panel por entidad federativa, ni controla por la posibilidad de causalidad inversa y/o sesgos generados por variables omitidas en el modelo. 
El presente artículo tiene un enfoque empírico y emplea datos de panel por entidad federativa para estudiar el impacto de la IED sobre la productividad laboral. Dicho enfoque es similar al de Baiashvili y Gattini (2020) y al que han usado en otros estudios a nivel estatal o por país para estudiar algunos determinantes de la productividad, entre los que se encuentran Belorgey et al. (2006), Bourlès et al. (2005 y 2007), Choudhry et al. (2016), Gust y Marquez (2004), Heshmati (2011), y Vergeer y Kleinknecht (2014).

La ventaja de emplear datos de panel radica en que si bien, como se observará en el documento, la productividad y su tasa de crecimiento a nivel nacional muestran poca variación, es posible encontrar una mayor heterogeneidad al comparar los datos por entidad federativa, y dicha variación es explotada para estimar los impactos de la tasa de crecimiento en la IED sobre la productividad laboral manufacturera. No existen estudios similares previos, al menos que sean de nuestro conocimiento.

\section{Productividad Laboral Manufacturera}

En esta sección se presentan dos diferentes medidas de productividad. La primera corresponde al Índice de Productividad Laboral de las Manufacturas (IPLM) estimado y publicado por INEGI, el cual se define como la razón entre el índice de volumen físico de la producción total y el índice de horas trabajadas en el sector manufacturero. Cabe mencionar que ambos índices se elaboran con información de la Encuesta Mensual de la Industria Manufacturera (EMIM). La EMIM contiene información de 240 clases industriales, por lo que resulta un instrumento estadístico de gran relevancia para medir el cambio en la productividad laboral, ya que es representativo para toda la industria manufacturera, y presenta periodicidad mensual a partir de enero de 2007 (los IPLM se publican de manera trimestral). Asimismo, su construcción conlleva un tratamiento cuidadoso que considera los precios de los productos y la diversidad de estos dentro de cada clase industrial. No obstante, vale la pena señalar que el IPLM no permite comparar los niveles de productividad entre entidades ya que es un índice.

La segunda medida es el Producto Medio por Trabajador (PMT) que también tiene como base la EMIM, y que se calculó para fines de este documento para contar con información 
sobre las diferencias en los niveles de productividad entre entidades federativas. Dicha medida, explota los datos abiertos de la Encuesta Mensual de la Industria Manufacturera disponibles a nivel entidad y sector de actividad económica en el portal del INEGI. ${ }^{4}$ Se hace uso del Valor de Producción de los Productos Elaborados, así como el Personal Ocupado Total. El valor de la producción se deflactó utilizando el Índice Nacional de Precios al Productor específico para las actividades secundarias sin petróleo.

Resulta relevante reconocer que existen diferencias sustanciales entre la medida de productividad que se genera a partir de los índices y la que es construida a partir de la información en niveles, a pesar de que ambas provienen de la misma encuesta. Como se mencionó anteriormente, uno de los insumos utilizados en el cálculo de los IPLM, es el Índice de Volumen Físico de la Producción Total, el cual conlleva un algoritmo complejo que implica tratar las observaciones desde nivel producto, estimar el precio de cada producto por medio del valor de la producción y el valor de ventas, así como obtener un índice de valor de la producción de los productos medidos en volumen y valor para las diversas clases de actividad. Es decir, el cálculo del índice de volumen físico de la producción, a diferencia de la medida de productividad estimada en niveles, toma en cuenta la diversidad de los productos dentro de cada clase de actividad industrial, así como el volumen de producción complementado con información de la Encuesta Industrial Anual, además de los ingresos por maquila, submaquila y remanufactura de materias primas y bienes semiprocesados propiedad de terceros, tanto nacionales como extranjeros. ${ }^{5}$ Por otro lado, la medida de productividad en niveles se estima por medio de un cálculo simplificado que involucra el valor de la producción de los productos elaborados y las horas trabajadas por el personal ocupado. Vale la pena mencionar que la información a nivel producto, que es la requerida para realizar el cálculo de los IPLM (y consecuentemente los valores a partir de los que se construyen dichos índices), se encuentra disponible dentro de INEGI únicamente a nivel nacional, no se encuentra con este nivel de desagregación por entidad federativa. A este nivel, únicamente

\footnotetext{
${ }^{4}$ Este es el máximo nivel de desagregación que se ofrece en el portal público del INEGI. Sin embargo, se debe considerar que en una función de producción técnicamente la mínima unidad de medida son los productos, los cuales posteriormente se agregan para conformar una clase de actividad, subrama, rama, subsector y sector.

${ }^{5}$ Para más detalles de este cálculo véase Anexo A y el documento “Cálculo de los índices de productividad laboral y Cumo 2015” de INEGI.
} 
se encuentra información a nivel subsector y no es posible encontrarla para todas las entidades federativas. Es por esto que ambas medidas están definitivamente reflejando ciertos aspectos de la productividad laboral de la industria manufacturera, pero es necesario considerar que existen diferencias entre ellas.

\subsection{Evolución de la Productividad Laboral de las Manufacturas a Nivel Nacional}

Durante el periodo 2007 - 2015 la productividad laboral medida por el IPLM creció 6.3 por ciento en promedio en México (Figura 1a), con una tasa de crecimiento compuesta promedio anual de 0.68 por ciento. Por su parte, el PMT, estimado con datos en niveles de la EMIM, registró un crecimiento total durante el periodo de 8.06 por ciento, para registrar una tasa de crecimiento compuesta promedio anual de 0.86 por ciento.

\subsection{Evolución de la Productividad Laboral de las Manufacturas en las Entidades}

Por otro lado, se estimó el promedio de las tasas de crecimiento anual de la productividad laboral de las manufacturas entre las entidades federativas de México para el periodo 20072015. ${ }^{6}$ Estas cifras muestran que la productividad laboral medida por el IPLM creció 1.08 por ciento en promedio entre las entidades en el periodo de referencia (Figura 1b), el mayor incremento promedio sucedió en 2010 (5.04 por ciento), mientras la menor tasa promedio se observó en 2008 (-1.37 por ciento). Por su parte, en el mismo periodo de referencia la variación de la productividad laboral de las entidades federativas, medida por el PMT, mostró en promedio un crecimiento del 1.28 por ciento, donde, de igual forma en el 2010, se observó la tasa anual más alta (6.88 por ciento) y en 2013 la tasa más baja (-1.12 por ciento). Resulta relevante observar que el promedio anual de las tasas de crecimiento compuestas de la productividad del trabajo en las manufacturas en las dos medidas utilizadas sigue un comportamiento consistente en general en la dirección de sus variaciones. Sin embargo, esto debe tomarse con reservas debido a que cada medida tiene particularidades en la información que contiene y esto se refleja en el hecho de que existen casos en los que algunas entidades

\footnotetext{
${ }^{6}$ Esta tasa de productividad promedio asume que todas las entidades tienen la misma importancia, ya que no son ponderadas con algún factor en especial.
} 
reflejan un cambio en la productividad en sentido opuesto dependiendo la medida utilizada, o difieren en las magnitudes de variación en el periodo.

Figura 1a

Evolución de la Productividad Laboral de las Manufacturas a Nivel Nacional, 2007-2015

Índice 2012=100, promedio anual

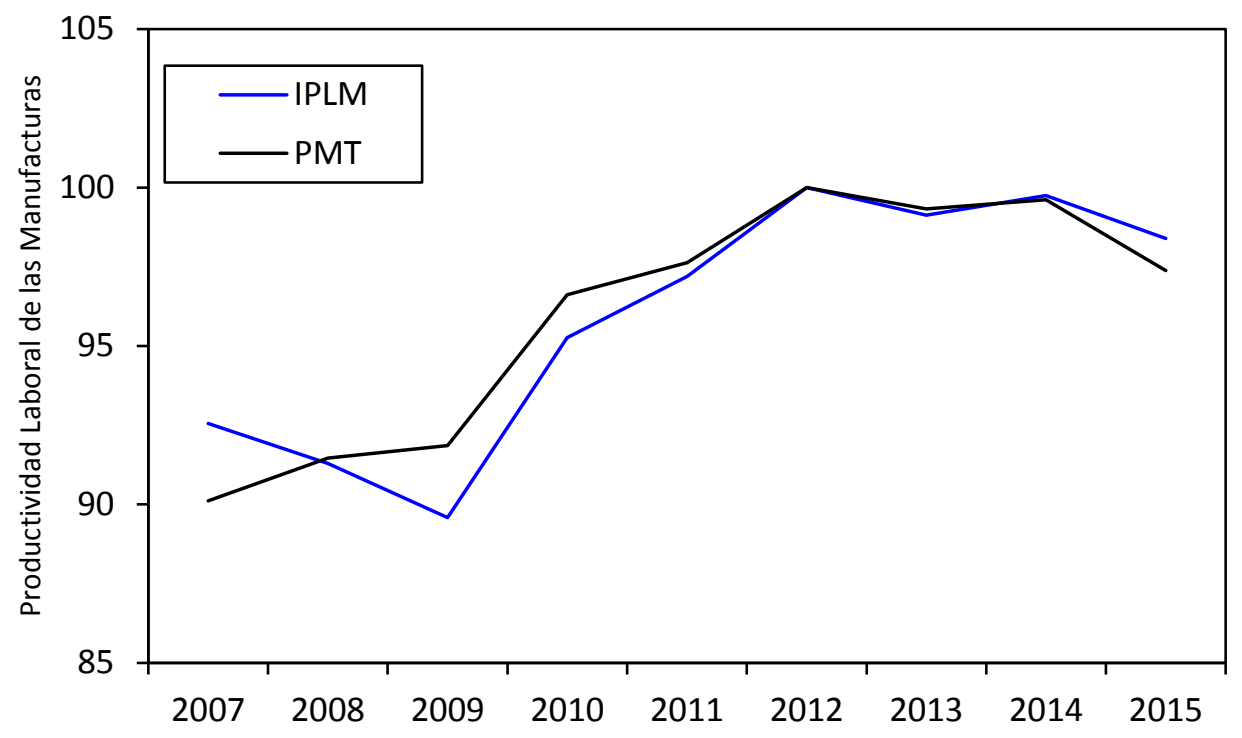

Figura 1b

Evolución del crecimiento promedio de la Productividad Laboral de las Manufacturas en las Entidades Federativas, 2008-2015

Tasas de crecimiento promedio (porcentaje)

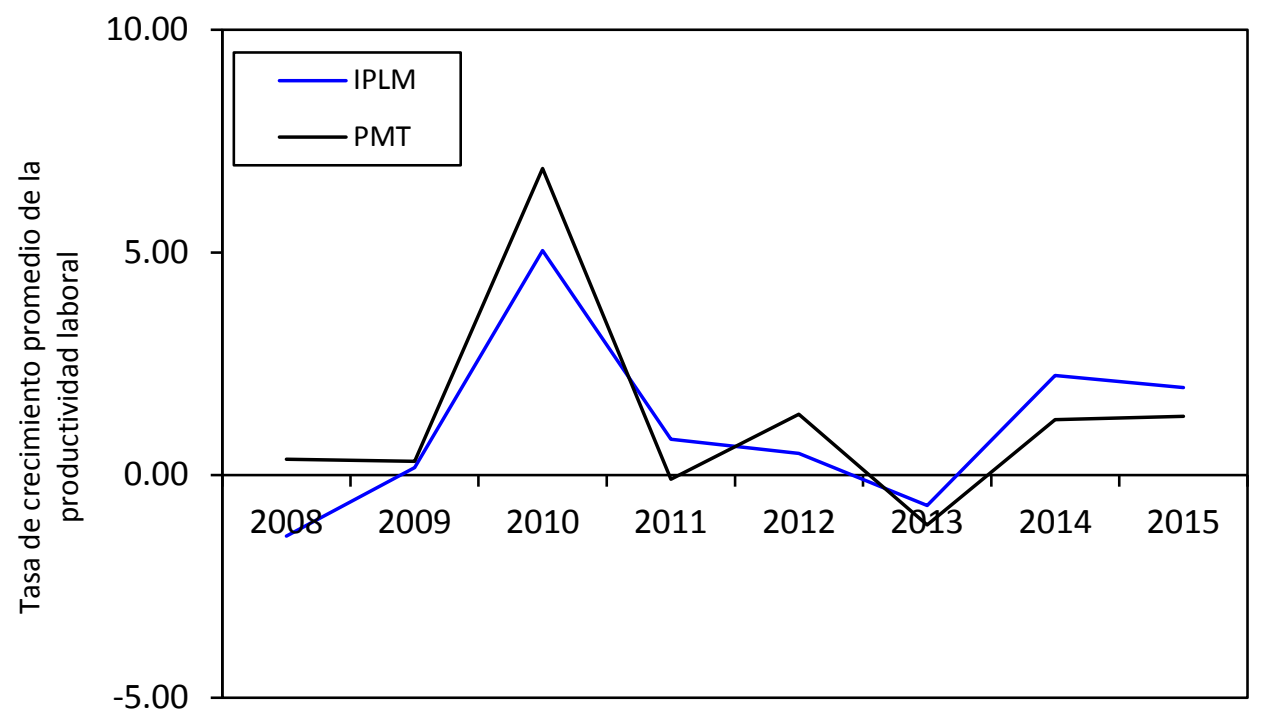

Fuente: Estimaciones propias con base en información del INEGI. 
La Figura 2 muestra el cambio en la productividad laboral de las manufacturas en las entidades del país, utilizando las dos medidas de productividad estimadas, durante el periodo antes mencionado. Dicho cambio está estimado por medio de la tasa de crecimiento compuesta promedio anual por entidad.

Figura 2

Cambio en la Productividad Laboral, 2007 - 2015

Tasa de crecimiento promedio anual compuesta (porcentaje)

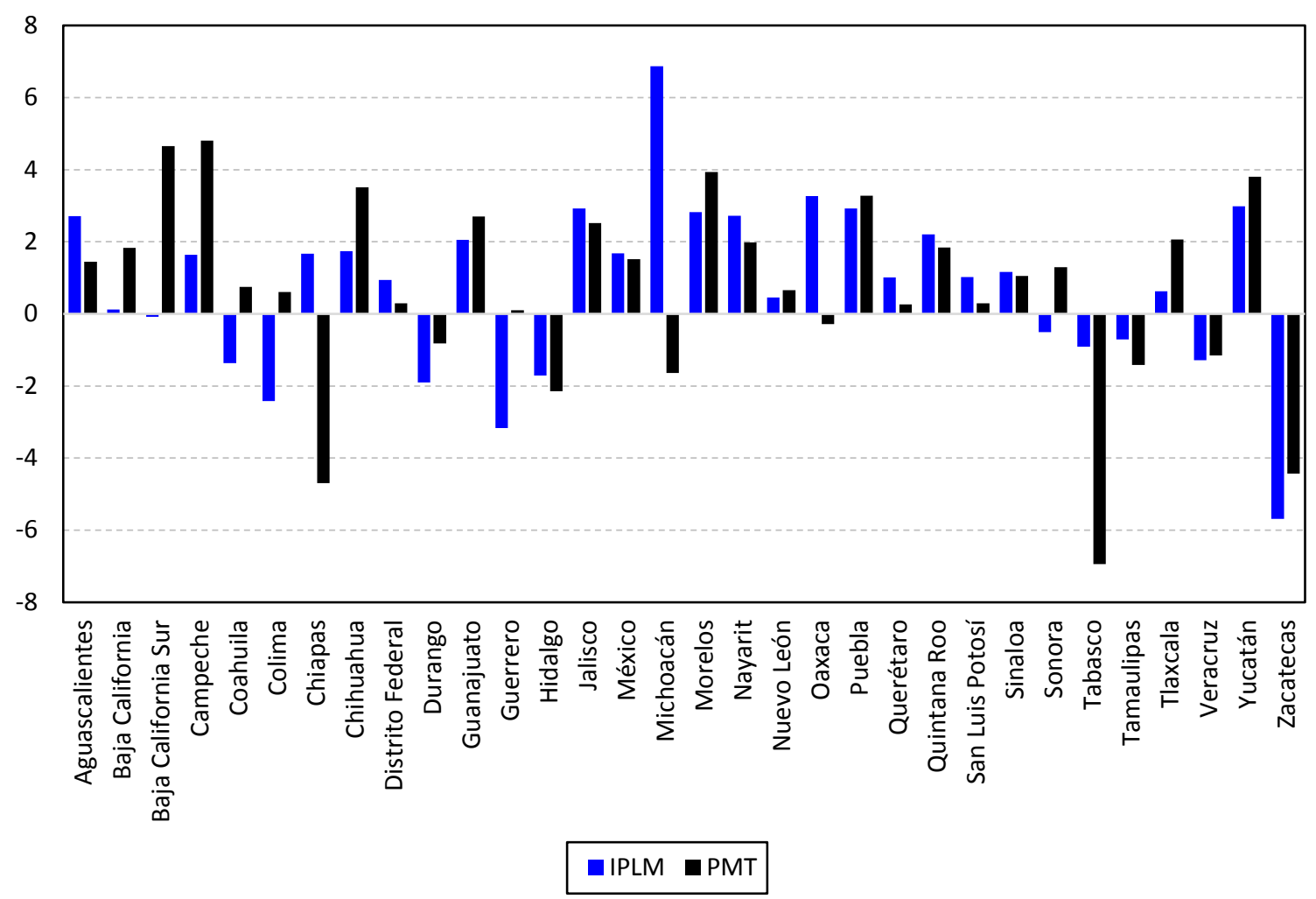

Fuente: Estimaciones propias con base en información del INEGI.

Se puede observar que para las dos medidas utilizadas existe cierta variabilidad en la evolución de la productividad laboral a nivel entidad federativa para el periodo 2007-2015, pero en general el cambio en la productividad entre ambas series se dio en el mismo sentido. Para la productividad laboral estimada con el IPLM la tasa compuesta media anual de crecimiento oscila en un rango entre -5.69 y 6.87 por ciento. Por un lado, existen entidades que registraron una disminución importante en sus niveles de productividad laboral, tales 
como Zacatecas (-5.69 por ciento), Guerrero (-3.17 por ciento), Colima (-2.42 por ciento) y Durango (-1.90 por ciento), mientras que por otro lado observamos estados como Michoacán (6.87 por ciento), Oaxaca (3.26 por ciento), Yucatán (2.98 por ciento), Puebla (2.92 por ciento) y Jalisco (2.92 por ciento) en los cuales se observó un incremento relativamente elevado en la productividad laboral.

Las tasas de crecimiento compuestas del PMT presentan un ordenamiento distinto de las entidades. De acuerdo con esta medida los estados que presentaron un menor crecimiento fueron Tabasco (-6.94 por ciento), Chiapas (-4.70 por ciento), Zacatecas (-4.43 por ciento), Hidalgo (-2.15 por ciento) y Michoacán (-1.64 por ciento), mientras las entidades que más crecieron a tasa anual fueron Campeche (4.80 por ciento), Baja California Sur (4.65 por ciento), Morelos (3.93 por ciento), Yucatán (3.81 por ciento) y Chihuahua (3.51 por ciento). La diferencia entre ambas medidas de productividad laboral también se manifiesta cuando se analiza la variación acumulada durante el periodo de análisis (Cuadro1). Si bien la variación promedio de todas las entidades es similar (8.95 vs 8.76), al analizar por entidades se observa en algunos casos diferencias marcadas, e incluso existen entidades como Baja California Sur, Chiapas, Guerrero y Michoacán que presentan signos contrarios.

\subsection{Productividad Laboral Relativa}

Como hemos observado hasta ahora, el comportamiento de la productividad laboral de la industria de las manufacturas entre las entidades es sumamente heterogéneo, sin embargo, resulta de utilidad conocer cómo dicho comportamiento se compara con el mostrado a nivel nacional. Para dicho fin se consideró relevante el análisis de la productividad laboral relativa de las manufacturas que presentan las distintas entidades, respecto al nivel nacional al inicio y al final del periodo de estudio. Para el PMT la productividad relativa se estima con el cociente del nivel que presenta cada entidad entre el respectivo valor a nivel nacional. Valores mayores a la unidad indican que la entidad analizada cuenta con una productividad superior al valor nacional, en contraste niveles inferiores a uno muestran un nivel menor al nacional. ${ }^{7}$

\footnotetext{
${ }^{7}$ No es posible estimar la productividad laboral relativa para los IPLM, ya que esta medida se expresa en índices, y no es comprable entre series.
} 


\section{Cuadro 1}

Variación total en la Productividad Laboral, 2007-2015

Por ciento

\begin{tabular}{|c|c|c|}
\hline \multirow[b]{2}{*}{ Entidad } & \multicolumn{2}{|c|}{ Medidas de productividad laboral } \\
\hline & IPLM & PMT \\
\hline Aguascalientes & 27.2 & 13.8 \\
\hline Baja California & 1.1 & 17.8 \\
\hline Baja California Sur & -0.7 & 50.6 \\
\hline Campeche & 15.8 & 52.5 \\
\hline Coahuila & -11.6 & 7.0 \\
\hline Colima & -19.8 & 5.6 \\
\hline Chiapas & 16.0 & -35.1 \\
\hline Chihuahua & 16.8 & 36.4 \\
\hline Distrito Federal & 8.8 & 2.7 \\
\hline Durango & -15.9 & -7.1 \\
\hline Guanajuato & 20.0 & 27.1 \\
\hline Guerrero & -25.2 & 0.9 \\
\hline Hidalgo & -14.4 & -17.8 \\
\hline Jalisco & 29.6 & 25.1 \\
\hline México & 16.2 & 14.5 \\
\hline Michoacán & 81.9 & -13.8 \\
\hline Morelos & 28.5 & 41.5 \\
\hline Nayarit & 27.3 & 19.3 \\
\hline Nuevo León & 4.2 & 6.1 \\
\hline Оахаса & 33.5 & -2.5 \\
\hline Puebla & 29.6 & 33.7 \\
\hline Querétaro & 9.5 & 2.4 \\
\hline Quintana Roo & 21.7 & 17.8 \\
\hline San Luis Potosí & 9.6 & 2.7 \\
\hline Sinaloa & 11.0 & 9.9 \\
\hline Sonora & -4.4 & 12.3 \\
\hline Tabasco & -7.9 & -47.7 \\
\hline Tamaulipas & -6.2 & -12.1 \\
\hline Tlaxcala & 5.8 & 20.2 \\
\hline Veracruz & -11.0 & -9.9 \\
\hline Yucatán & 30.2 & 40.0 \\
\hline Zacatecas & -41.0 & -33.5 \\
\hline Tasa prome dio & 8.95 & 8.76 \\
\hline
\end{tabular}

Fuente: Estimaciones propias con base en información del INEGI. 
La productividad laboral relativa se presenta en la Figura 3, la cual muestra la distribución geográfica de las brechas de productividad de las entidades respecto al nivel nacional para los años 2007 y 2015. Las entidades con colores más intensos en el rango son aquellas que poseen una productividad relativa más grande respecto al nivel nacional y viceversa.

De acuerdo con el PMT, en el periodo comprendido entre el año 2007 y el año 2015, la distribución geográfica y los niveles de la productividad laboral relativa se mantuvieron prácticamente iguales, como es posible observar en los mapas correspondientes de la Figura 3. Las entidades con mayor productividad respecto a la nacional en 2007 fueron Oaxaca (6.38), Tabasco (6.25), Chiapas (3.47) y Veracruz (2.85). La mayoría de estos estados se mantuvieron con una alta productividad relativa para el año 2015, (Oaxaca con un nivel de 5.76, Tabasco con 3.02, Veracruz con 2.37 y Chiapas con 2.08). Por otro lado, las entidades federativas con más bajos coeficientes de productividad laboral relativa al nivel nacional en el inicio del periodo fueron Chihuahua (0.11), Campeche (0.12) y Baja California (0.20). Mientras en 2015 dichos estados vuelven a aparecer como los que presentan una menor productividad relativa (Chihuahua (0.14), Campeche (0.17) y Baja California con (0.22). Esta distribución geográfica presenta una fuerte concentración de entidades con productividad laboral relativa alta en la región Sur. Dada la especialización de la región antes mencionada en el sector económico de extracción de petróleo, parecen existir indicios de que los subsectores de las manufacturas asociados a la fabricación de derivados del petróleo y del carbón han mantenido una alta productividad respecto a otros subsectores dentro de la industria manufacturera en varias de estas entidades durante el periodo analizado. 


\section{Figura 3}

Productividad Laboral Relativa

Cociente entre la de productividad laboral manufacturera de la entidad y la del país

PMT, 2007

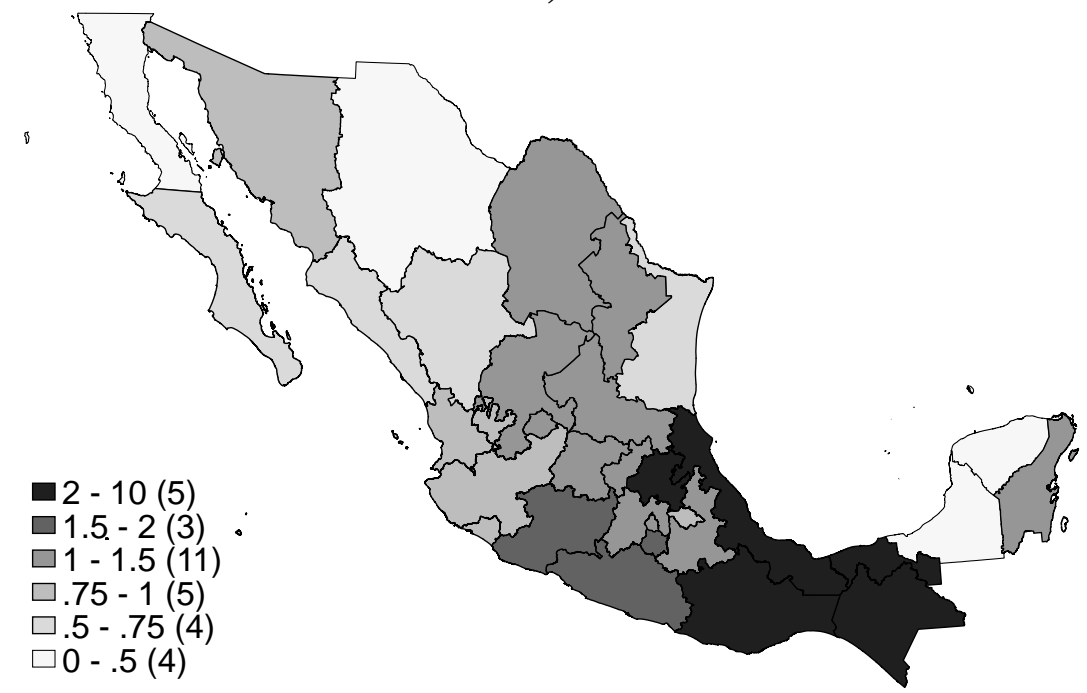

PMT, 2015

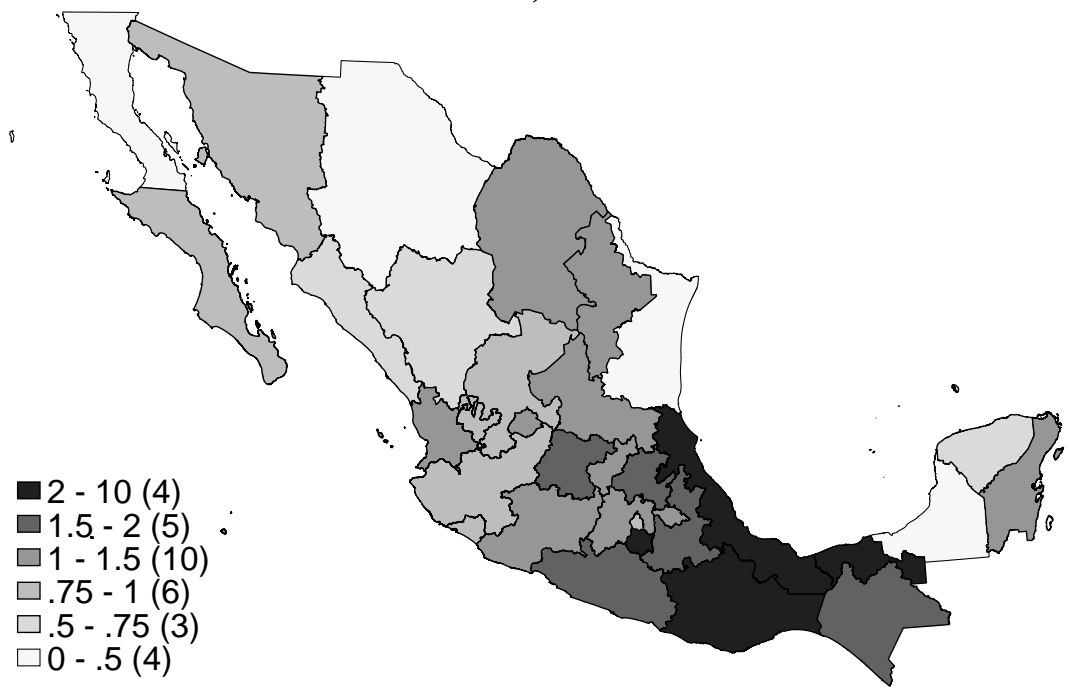

Fuente: elaboración propia con base en datos de la EMIM. 


\section{Figura 4}

Correlación en el Ordenamiento de los Niveles de Productividad, 2007-2015

PMT

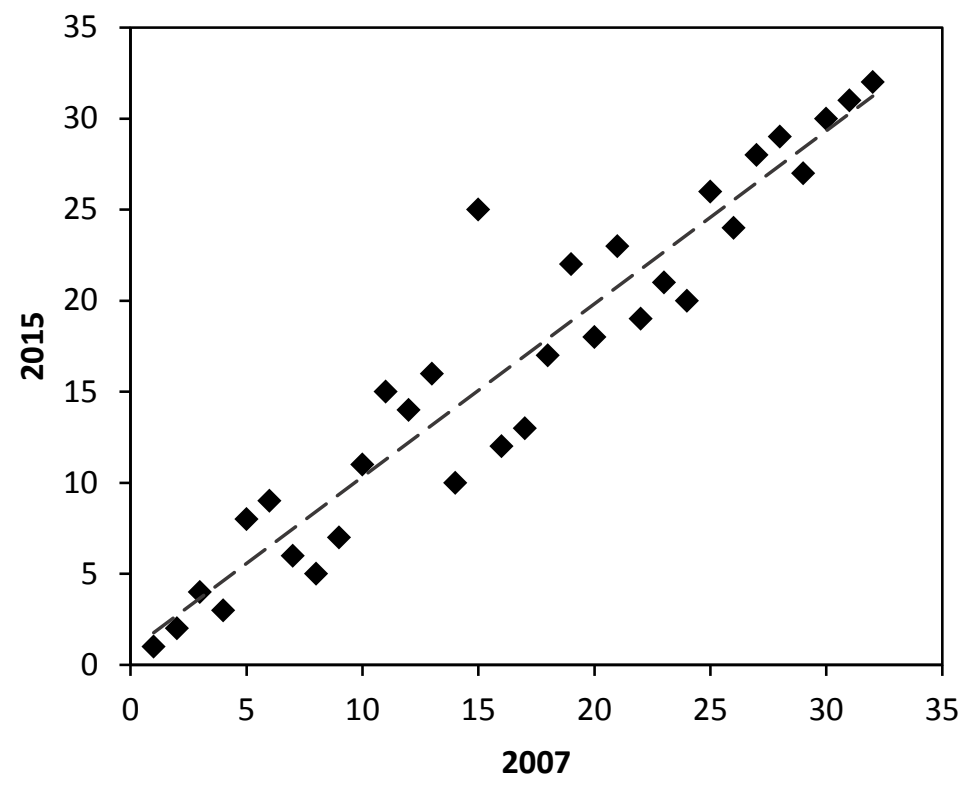

Fuente: elaboración propia con base en datos de la EMIM.

La Figura 4 muestra la correlación entre el ordenamiento (ranking) de los niveles de productividad laboral manufacturera de las entidades federativas en 2007 contra sus respectivos niveles en 2015, para el PMT. En dicha medida de producto por trabajador se puede observar que se guardó un ordenamiento similar en ambos cortes temporales, es decir, se puede sospechar que los niveles de variación de la productividad en el periodo de estudio no fueron lo suficientemente heterogéneos como para cambiar el ordenamiento general de las entidades de México, al observarse un movimiento en "bloque". El coeficiente de correlación entre el ranking de PMT en 2007 y en 2015 es de 0.95, lo que confirma que, si bien se ha registrado una especie de efecto catch up, este no ha sido tan grande como para alterar el ordenamiento en los niveles de productividad.

Para finalizar esta sección, resulta relevante recordar que el uso del IPLM como medida de productividad ofrece bondades importantes cuando se está estudiando el cambio de la productividad a través del tiempo, ya que como se comentó al inicio de esta sección, la construcción de dicho índice se realiza de manera más fina respecto a una variable autoconstruida, ya que toma en cuenta detalles relevantes de los precios de los productos que 
componen el índice y reconoce la diversidad de las clases de actividad industrial entre entidades. Por otro lado, la construcción de la PMT nos permite analizar y dimensionar los niveles de productividad, y realizar comparaciones entre entidades en un mismo periodo temporal, con la salvedad de que realiza una contabilidad menos precisa de la productividad laboral en el tiempo toda vez que el ajuste de la variación en precios se realiza por medio del índice nacional de precios al productor sin petróleo. Por lo tanto, se considera que el estudio de estas dos variables en su conjunto para robustecer el panorama de la productividad laboral de la industria manufacturera en México.

\section{Modelo Econométrico}

Para identificar el efecto de la IED sobre la productividad laboral en la industria manufacturera de las entidades del país se emplea como punto de partida el modelo utilizado por Baiashvili y Gattini (2020) y Gust y Marquez (2004): ${ }^{8}$

$$
\Delta y_{i t}=\rho \Delta y_{i t-1}+\theta X_{i t-1}+\gamma I E D_{i t-1}+\delta_{t}+\varepsilon_{i}+\mu_{i t}
$$

Donde el término $\Delta y_{i t}$ es la tasa de crecimiento de la productividad laboral del sector manufacturero en la entidad i durante el tiempo t, ${ }^{9} I E D_{i t-1}$ es la inversión extranjera directa manufacturera como porcentaje del PIB de dicho sector en la entidad i rezagada un periodo, $X_{i t-1}$ es un vector de variables de control (con un rezago) que influyen sobre el crecimiento económico, las variables se rezagan un periodo con la finalidad de atenuar posible problemas de causalidad inversa (Baiashvili y Gattini 2020; Gust y Marquez, 2004). Por su parte, $\delta_{t}$ y $\varepsilon_{i}$ representan efectos fijos de tiempo y entidad federativa respectivamente, mientras que $\mu_{i t}$ corresponde al término de error. Siguiendo la práctica común en la literatura se agrega la variable dependiente rezagada (Belorgey et al. 2006; Bourlès et al. 2005 y 2007; Dimelis y Papaioannau 2010; Vergeer y Kleinknecht, 2014). La variable rezagada de la tasa de crecimiento se incorpora debido a la persistencia temporal de la estructura productiva en las

\footnotetext{
${ }^{8}$ Belorgey et al. (2006), Bourlès et al. (2005 y 2007) han empleado especificaciones similares para analizar los efectos de la inversión como porcentaje del PIB y la inversión en el sector telecomunicaciones, si bien en dichos estudios se utilizan los valores contemporáneos de las variables explicativas.

${ }^{9}$ Dado que muchas de las variables empleadas en el análisis están disponibles con frecuencia anual se decidió calcular el promedio por año de dicha variable.
} 
entidades federativas, en la IED y en la infraestructura física disponible que, de no incorporarse en el modelo, arrojaría estimadores sesgados e ineficientes en los determinantes de la tasa de crecimiento de la productividad laboral. Bourlès et al. (2005 y 2007) argumentan que la incorporación de dicho término permite la posibilidad de capturar un impacto continuo de las variables explicativas en la productividad laboral, lo cual es relevante ya que el impacto de variables como la Infraestructura y la IED pueden reflejarse durante varios años y no solo en un punto en el tiempo. Un coeficiente positivo en la variable dependiente rezagada indica que el impacto de largo plazo generado por las otras variables explicativas es mayor al impacto de corto plazo. Y lo contrario sucede cuando el citado coeficiente es negativo. En principio, el coeficiente puede tomar cualquier signo con la única restricción de ser menor a uno (en términos absolutos).

La IED fue modelada como un determinante endógeno de la productividad debido a que la causalidad entre esta variable y la productividad podría darse en ambos sentidos. En efecto, un incremento en la productividad podría ser generado por la IED a través de cadenas de producción, imitación de procesos productivos, creación de nuevos productos y procesos, y capacitación en el empleo (Zhao y Zhang, 2010), o porque permite la creación de empleo y acumulación de capital físico (Mitze y Özyurt, 2014). No obstante, también podría ocurrir que aquellas entidades manufactureras que presentan un elevado nivel de productividad laboral tiendan a atraer mayores flujos de IED. Adicionalmente, existe la posibilidad de variables no observadas u omitidas en el modelo que estén correlacionadas en forma positiva o negativa con la IED y que generen una sobreestimación o subestimación de su impacto, como podrían ser los niveles de desplazamiento de inversión privada domestica (Fillat y Woerz, 2011) o el hecho de que algunas de las empresas transaccionales restrinjan la difusión de tecnologías y la innovación (Zhao y Zhang, 2010).

El vector de variables de control contiene diversos factores que usualmente se emplean en la literatura y que influyen sobre el crecimiento económico. En específico, se utilizan controles de capital humano, cambios en el uso del factor trabajo, capital físico o infraestructura, grado de apertura comercial y de desarrollo financiero e inflación. El capital humano se mide a través del porcentaje de la población ocupada en las manufacturas con 13 o más años de escolaridad, y se introduce ya que dicho factor mejora las capacidades laborales del 
individuo. Asimismo, la población altamente educada potencializa la adaptación de las nuevas tecnologías, lo que a su vez permite un mayor crecimiento de la productividad (Harris, 2002).

Siguiendo a Mitra et al. (2002) se incluye como variable de capital físico un índice que aproxima la dotación de infraestructura por entidad federativa. Dicho índice fue calculado por medio de la técnica de componentes principales y toma en cuenta variables de infraestructura carretera, férrea, de transporte, de telecomunicaciones, social, financiera, eléctrica, turística y financiera. ${ }^{10}$ Dado que los índices formados mediante componentes principales pueden tomar valores negativos, se decidió normalizar esta variable en un rango de cero a uno. La construcción de este índice permite considerar 9 variables de infraestructura en un solo índice, por lo que evita problemas de multicolinealidad entre dichas variables y ahorra grados de libertad que son muy importantes en la implementación de modelos dinámicos de panel estimados por medio del método generalizado de momentos, debido a la gran cantidad de instrumentos que estos emplean. En este sentido, el citado índice podría representar una mejora con respecto a estudios que solamente emplean una o dos variables de infraestructura en su modelo (tradicionalmente kilómetros de carretera o de vías férreas, y densidad telefónica).

Los cambios en el uso del factor trabajo son capturados por medio de la tasa de crecimiento del empleo, la cual fue estimada empleando datos de la EMIM. Esta variable ha sido empleada en diversos documentos como factor de control (Belorgey et al. 2006; Bourlès et al. 2005 y 2007). ${ }^{11}$ Como medida del grado de apertura comercial se emplean las exportaciones manufactureras como proporción del PIB de ese sector, dicha variable se introduce en el modelo ya que una mayor apertura comercial incentiva la especialización, el uso de las ventajas comparativas y la mayor eficiencia (Dimelis y Papaioannau, 2010). Asimismo, se argumenta que el comercio internacional es un canal importante en el uso y adaptación de la tecnología (Feenstra et al, 1992; Markusen, 1999). Por su parte, el grado de

\footnotetext{
${ }^{10}$ Para conocer detalles de la construcción del índice véase Anexo B.

${ }^{11}$ Otros estudios que buscan medir el impacto sobre la productividad laboral total utilizan la tasa de crecimiento poblacional. Ver por ejemplo Alfaro et al. (2004).
} 
desarrollo financiero es medido por la proporción de la cartera de crédito total como porcentaje del PIB. De acuerdo a Alfaro et al. (2004) el desarrollo financiero puede afectar el crecimiento económico e incluso podría influir sobre los efectos de IED en el crecimiento económico. Finalmente, siguiendo a estudios como Alfaro et al. (2004), Baiashvili y Gattini (2020) y Carkovic y Levine (2005) se incluyó la inflación por entidad federativa como variable de control.

En el cuadro 2 se presenta una descripción de cada una de las variables utilizadas en el modelo, así como sus unidades de medida en las que se incorporan en las regresiones y las fuentes de información.

\section{Cuadro 2}

Variables del Modelo

\begin{tabular}{|c|c|c|}
\hline Variable & Unidad de medida & Fuente \\
\hline IPLM & Tasa de Crecimiento & EMIM \\
\hline PMHT & Tasa de Crecimiento & EMIM \\
\hline Es colaridad (Población con 13 o más años) & Proporción (Logaritmos) & ENOE \\
\hline IED & Porcentaje del PIB Manufacturero & INEGI \\
\hline Empleo EMIM & Tasa de Crecimiento & EMIM \\
\hline $\begin{array}{l}\text { Infraestructura (Índice de Componentes } \\
\text { Principales) }{ }^{1 /}\end{array}$ & Índice normalizado (Logaritmos) & INEGI \\
\hline Exportaciones & Proporción del PIB Manufacturero (Logaritmos) & INEGI \\
\hline Crédito Total & Porcentaje del PIB (Logaritmos) & Banco de México/INEGI \\
\hline Inflación & Tasa de Crecimiento del IPC & INEGI \\
\hline
\end{tabular}

$\overline{1 / \text { Las fuentes y unidades de medida de las variables utilizadas para construir el Índice de Infraestructura se }}$ pueden consultar en el Cuadro B1 del Anexo B.

Nota: Todas las variables explicativas entran rezagadas un periodo en especificación econométrica.

Fuente: Estimaciones propias.

Para estimar la ecuación 1 se utiliza el método general de momentos empleando tanto la propuesta de Arellano y Bond (1991), MGM-A como la de Blundell y Bond (1998), MGMBB. Sin embargo, se eligen como estimaciones principales las calculadas por el MGM-BB, ya que generan estimadores más robustos que los obtenidos por medio del estimador MGM$\mathrm{AB}$, cuando la varianza de los efectos del panel en relación a $\mu_{i t}$ es relativamente grande o cuando el proceso autorregresivo es persistente en el tiempo. ${ }^{12}$ Esta estimación permite

12 El método de Blundell y Bond (1998) impone condiciones adicionales de momentos en las cuales las diferencias rezagadas son usadas como instrumentos en la ecuación en niveles, además de las condiciones de momento en donde se usan las variables rezagadas en niveles como instrumentos de la ecuación en diferencias. 
controlar por la endogeneidad de la IED empleando variables rezagadas como instrumentos, así como por la dependencia intertemporal de la variable explicada al incluir un rezago de la misma. Además, permite incorporar efectos fijos por entidad federativa para así controlar por variables no observables que no cambian en el tiempo (Baltagi y Rich, 2005).

Los modelos se estiman asumiendo a la IED $_{\mathrm{t}-1}$ como variable endógena (es decir se permite que esta variable este correlacionada con el error en $\mathrm{t}$ y en periodos previos) y al resto de las variables de control se asume que son estrictamente exógenas. El MGM-AB aplica diferencias a la primera ecuación y utiliza las siguientes condiciones de momentos:

$$
\begin{gathered}
E\left[\Delta y_{i, t-s} \cdot\left(\mu_{i, t}-\mu_{i, t-1}\right)\right]=0 \text { para s }>1 \mathrm{t}=3, \ldots \mathrm{T} . \\
E\left[I E D_{i,(t-1)-s} \cdot\left(\mu_{i, t}-\mu_{i, t-1}\right)\right]=0 \text { para } \mathrm{s}>1 \mathrm{t}=3, \ldots \mathrm{T} . \\
E\left[X_{i,(t-1)-s} \cdot\left(\mu_{i, t}-\mu_{i, t-1}\right)\right]=0 \text { para todo s } \mathrm{y} .
\end{gathered}
$$

Las condiciones adicionales de momento impuestas por el MGM-BB sobre la ecuación en niveles (ecuación 1 sin tomar diferencias) son:

$$
\begin{gathered}
E\left[\left(\Delta y_{i, t-s}-\Delta y_{i, t-s-1}\right) \cdot\left(\varepsilon_{i}+\mu_{i, t}\right)\right]=0 \text { para s }=1 . \\
E\left[\left(I E D_{i,(t-1)-s}-I E D_{i,(t-1)-s-1}\right) \cdot\left(\varepsilon_{i}+\mu_{i, t}\right)\right]=0 \text { para } \mathrm{s}=1 . \\
E\left[\left(X_{i,(t-1)-s}-X_{i,(t-1)-s-1}\right) \cdot\left(\varepsilon_{i}+\mu_{i, t}\right)\right]=0 \text { para } \mathrm{s}=1 .
\end{gathered}
$$

Cabe señalar que la calidad de los estimadores obtenidos a través de la técnica generalizada de momentos depende de dos factores (Arellano y Bond, 1991): i) que el término de error de la ecuación en primera diferencia no este serialmente correlacionado; ii) la validez de la matriz de instrumentos. Por esta razón se realizan las pruebas de correlación serial de Arrellano y Bond y de sobre identificación de instrumentos de Sargan.

Las estimaciones son realizadas empleando el estimador de dos pasos aplicando errores estándar robustos. Dichos estimadores tienen mayor precisión que los estimadores GMM de un solo paso, si bien en necesario aplicar una corrección en los errores estándar ya que estos tienden a estar subestimados (Arellano y Bond, 1991). ${ }^{13}$

\footnotetext{
${ }^{13}$ Se aplica la corrección propuesta por Windmeijer (2005) e implementada por el software STATA.
} 


\section{Estadísticas Descriptivas}

En el Cuadro 3 se puede observar la estadística descriptiva de las variables económicas que son empleadas como variables explicativas en el modelo econométrico, se presentan los estadísticos correspondientes al promedio, fecha inicial y final del periodo bajo análisis. ${ }^{14} \mathrm{Es}$ posible observar que las tasas anuales de crecimiento del empleo registraron en el agregado variaciones muy pequeñas en el periodo de análisis. Por su parte, los flujos de IED como proporción del PIB son mayores en el año 2015 que en el 2008, si bien dicha variable cambia constantemente en el tiempo y entre entidades, el valor promedio durante todo el periodo fue de $8.8 \%$. Asimismo, se observa que la variable de infraestructura normalizada registra un valor más alto al finalizar el periodo de análisis, mientras que el porcentaje promedio de la población con 13 o más años de escolaridad se incrementó de 10.2 a 12.9 por ciento de 2008 a 2015. En referencia a la variable de desarrollo financiero se observa que dicha variable registra niveles promedio más elevados en 2015 comparado con 2008. Los datos también indican que las exportaciones promedio como proporción del PIB manufacturero aumentaron. En contraste, se observan niveles de inflación promedio por entidad federativa más bajos en 2015 en relación a los observados en 2008.

\section{Cuadro 3}

Estadísticos Descriptivos, 2008 y 2015

\begin{tabular}{|c|c|c|c|c|c|c|}
\hline & \multicolumn{2}{|c|}{2008} & \multicolumn{2}{|c|}{2015} & \multicolumn{2}{|c|}{$2007-2015$} \\
\hline & Promedio & $\begin{array}{l}\text { Desviación } \\
\text { Estándar }\end{array}$ & Promedio & $\begin{array}{l}\text { Desviación } \\
\text { Estándar }\end{array}$ & Promedio & $\begin{array}{c}\text { Desviación } \\
\text { Estándar }\end{array}$ \\
\hline IED $^{1 /}$ & 0.055 & 0.053 & 0.096 & 0.158 & 0.088 & 0.144 \\
\hline Infraestructura ${ }^{2 /}$ & 0.264 & 0.155 & 0.307 & 0.159 & 0.281 & 0.160 \\
\hline Escolaridad $^{3 /}$ & 0.102 & 0.045 & 0.129 & 0.049 & 0.113 & 0.045 \\
\hline Exportaciones ${ }^{1 /}$ & 0.968 & 0.940 & 1.132 & 0.964 & 1.049 & 1.017 \\
\hline Cartera de Créditos ${ }^{4 /}$ & 0.058 & 0.054 & 0.082 & 0.077 & 0.069 & 0.061 \\
\hline Inflación & 0.050 & 0.006 & 0.026 & 0.005 & 0.040 & 0.010 \\
\hline Empleo EMIM $^{5 /}$ & -0.015 & 0.062 & 0.017 & 0.134 & 0.007 & 0.078 \\
\hline
\end{tabular}

1/ Proporción del PIB Manufacturero 2/Índice normalizado 3/Proporción 4/Proporción del PIB de la Entidad 5/Tasa de crecimiento Fuente: Estimaciones propias.

\footnotetext{
${ }^{14}$ Se presenta 2008 en lugar de 2007, ya que algunas variables están medidas en tasa de crecimiento.
} 
Las estadísticas descriptivas agregadas de las variables recién analizadas muestran en algunos casos poca variación durante el periodo temporal estudiado, sin embargo, es importante señalar que existe un alto grado de heterogeneidad una vez que se analizan los datos por entidad federativa (Cuadro 4). Por ejemplo, se encuentran entidades con proporciones elevadas de IED en relación al PIB manufacturero como Baja California Sur y Zacatecas, mientras que otros como Tabasco, Sinaloa, Durango y Chiapas cuentan con valores muy bajos. Asimismo, es posible observar una gran heterogeneidad en la infraestructura, por un lado, la Ciudad de México y Nuevo León cuentan con niveles muy elevados, por otro lado, entidades como Oaxaca y Chiapas muestran los menores índices de infraestructura. En referencia a las tasas de crecimiento del factor trabajo (empleo) se observa que, si bien los cambios en dicha variable no son tan grandes, sí existe heterogeneidad entre entidades (algunas mostrando tasas positivas y otras tasas negativas). En términos de capital humano también se observan diferencias significativas, la Ciudad de México, Tamaulipas, Nuevo León y Coahuila cuentan con proporciones más elevadas de la población con 13 o más años de escolaridad, mientras que las proporciones más bajas se observan en Guerrero, Oaxaca y Chiapas. En relación a las exportaciones como proporción del PIB se observa que los estados del norte del país como Chihuahua, Baja California y Tamaulipas presentan los mayores niveles. Por su parte, la variable indicativa de desarrollo financiero observa sus mayores niveles en Ciudad de México y Nuevo León. En contraste, los niveles más bajos de esta variable se registran en Tlaxcala, Guerrero y Chiapas. Finalmente, también se identifican ciertas diferencias en los niveles de inflación. 


\section{Cuadro 4}

Estadísticos Descriptivos por Entidad (2007-2015)

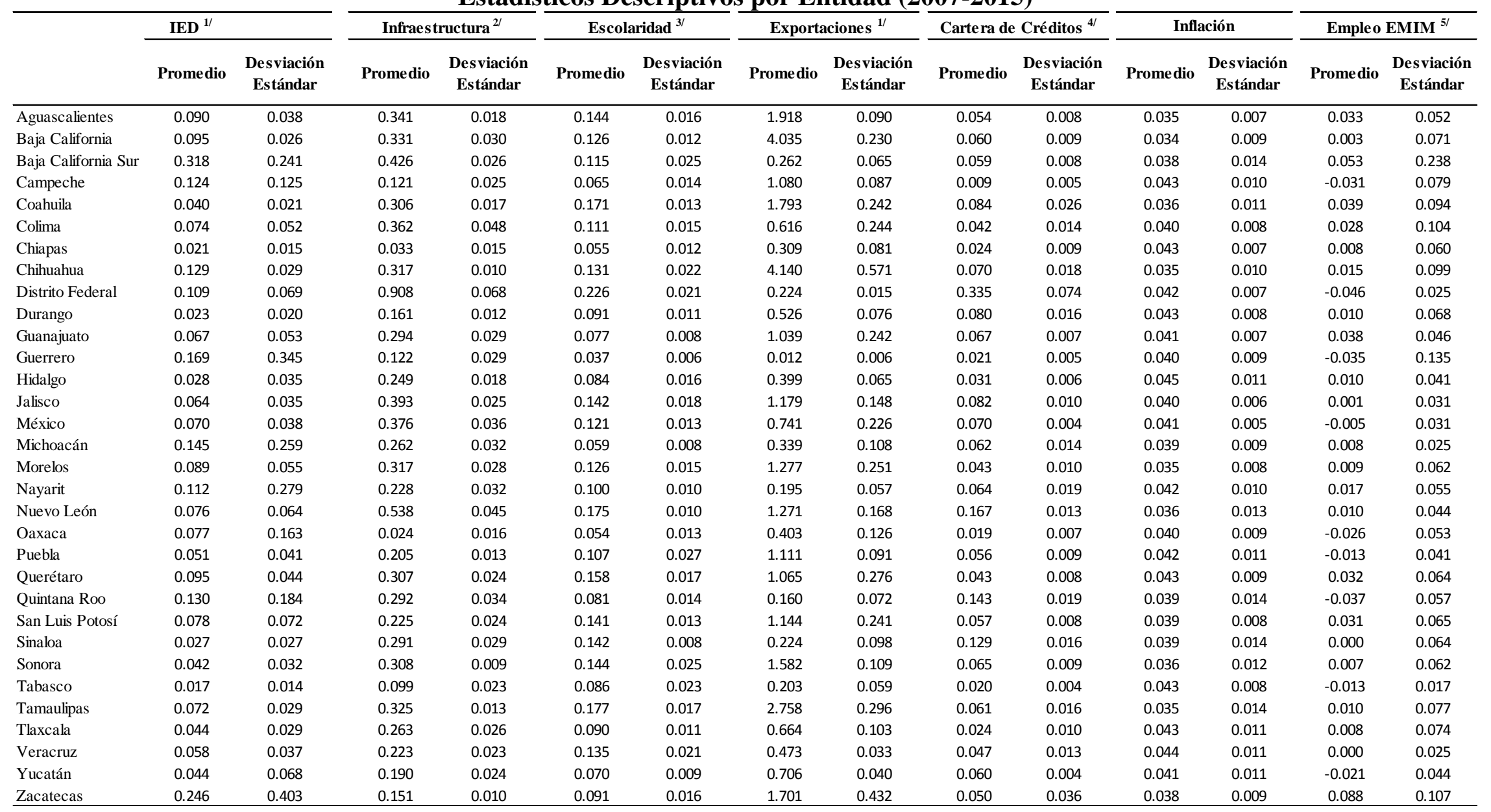

1/ Proporción del PIB Manufacturero 2/Índice normalizado 3/Proporción 4/Proporción del PIB de la Entidad 5/Tasa de crecimiento Fuente: Estimaciones propias. 


\section{Resultados}

La última columna del Cuadro 5 presenta las estimaciones de la ecuación 1 cuando se emplea IPLM para estimar la tasa de crecimiento de la productividad laboral (variable dependiente) y se utiliza el MGM-BB. Con la finalidad de verificar la robustez de los resultados y/o estabilidad del modelo se presentan diversas especificaciones en las primeras seis columnas. La primera columna muestra el modelo base en donde además de la IED se incluye la tasa de crecimiento del empleo. En las columnas 2 a la 6 se incluyen por separado los controles relacionados a la infraestructura, escolaridad, apertura comercial, inflación y grado de desarrollo financiero, en ese orden. ${ }^{15}$ Las interpretaciones se realizarán tomando el modelo más completo que aparece en la columna $7 .{ }^{16}$

Cabe señalar que todas las especificaciones econométricas satisfacen las pruebas de sobre identificación de Sargan, y de correlación serial de Arellano Bond mostrando que se rechaza la ausencia de correlación serial de primer orden, pero no se puede rechazar la ausencia de correlación de segundo orden. Lo anterior sugiere la correcta especificación de los modelos estimados. Asimismo, vale la pena recordar que la ecuación bajo análisis no puede ser estimada mediante mínimos cuadrados ordinarios o métodos tradicionales de panel como efectos fijos pues generaría estimadores sesgados, toda vez que la ecuación a estimar contiene un rezago de la variable dependiente. Sin embargo, debe aclararse que los coeficientes de los modelos dinámicos de panel reflejan efectos de corto plazo más que estimaciones de largo plazo.

\footnotetext{
${ }^{15}$ Para realizar las estimaciones se restringió el número de regazos a 3, tanto en la variable dependiente como en las variables explicativas, ya que un número muy elevado de instrumentos cuando el número de observaciones en el tiempo es limitado puede generar que las estimaciones de GMM sean sesgadas e ineficientes (Kiviet, 1995).

${ }^{16}$ Los resultados son similares si se reestima el modelo empleando como variable dependiente la productividad por hora de trabajo. Los resultados están disponibles bajo petición en caso de que así se requieran.
} 


\section{Cuadro 5}

Efectos de la IED sobre la Tasa de Crecimiento del IPLM (MGM-BB)

\begin{tabular}{|c|c|c|c|c|c|c|c|}
\hline & (1) & (2) & (3) & (4) & (5) & (6) & (7) \\
\hline Primer rezago IPML ${ }^{1 /}$ & $\begin{array}{c}0.037 \\
(0.097)\end{array}$ & $\begin{array}{c}0.038 \\
(0.099)\end{array}$ & $\begin{array}{c}0.026 \\
(0.079)\end{array}$ & $\begin{array}{c}0.014 \\
(0.072)\end{array}$ & $\begin{array}{l}0.063 * \\
(0.038)\end{array}$ & $\begin{array}{c}0.040 \\
(0.081)\end{array}$ & $\begin{array}{c}0.034 \\
(0.077)\end{array}$ \\
\hline $\mathrm{IED}^{2 /}$ & $\begin{array}{l}0.083 * \\
(0.050)\end{array}$ & $\begin{array}{l}0.086^{*} \\
(0.045)\end{array}$ & $\begin{array}{c}0.068 * * \\
(0.031)\end{array}$ & $\begin{array}{l}0.069 * \\
(0.042)\end{array}$ & $\begin{array}{l}0.097 * \\
(0.052)\end{array}$ & $\begin{array}{l}0.079 * \\
(0.047)\end{array}$ & $\begin{array}{l}0.078 * \\
(0.046)\end{array}$ \\
\hline Empleo EMIM $^{1 /}$ & $\begin{array}{l}-0.162 * \\
(0.092)\end{array}$ & $\begin{array}{c}-0.180^{* *} \\
(0.088)\end{array}$ & $\begin{array}{c}-0.200 * * \\
(0.090)\end{array}$ & $\begin{array}{c}-0.187^{* *} \\
(0.090)\end{array}$ & $\begin{array}{c}-0.206^{*} \\
(0.108)\end{array}$ & $\begin{array}{c}-0.183^{* *} \\
(0.085)\end{array}$ & $\begin{array}{c}-0.252^{* *} \\
(0.113)\end{array}$ \\
\hline Infraestructura $^{3 /}$ & & $\begin{array}{l}-0.001 \\
(0.020)\end{array}$ & & & & & $\begin{array}{c}0.001 \\
(0.048)\end{array}$ \\
\hline Escolaridad $^{3 /}$ & & & $\begin{array}{c}0.008 \\
(0.010)\end{array}$ & & & & $\begin{array}{c}0.027 \\
(0.031)\end{array}$ \\
\hline Exportaciones $^{3 /}$ & & & & $\begin{array}{l}-0.004 \\
(0.007)\end{array}$ & & & $\begin{array}{c}0.003 \\
(0.025)\end{array}$ \\
\hline Inflación ${ }^{3 /}$ & & & & & $\begin{array}{c}0.018 \\
(0.015)\end{array}$ & & $\begin{array}{c}0.014 \\
(0.012)\end{array}$ \\
\hline Cartera $^{3 /}$ & & & & & & $\begin{array}{l}-0.003 \\
(0.008)\end{array}$ & $\begin{array}{c}0.015 \\
(0.032)\end{array}$ \\
\hline Control de Endogeneidad & SI & SI & SI & SI & SI & SI & SI \\
\hline Sargan & 30.141 & 30.313 & 28.697 & 28.112 & 28.282 & 28.693 & 23.463 \\
\hline Sargan [p-value] & 0.815 & 0.808 & 0.862 & 0.880 & 0.875 & 0.863 & 0.969 \\
\hline Arellano-Bond (1) & -2.138 & -2.347 & 2.155 & -2.322 & -2.319 & -2.664 & -2.438 \\
\hline Arellano-Bond (1) [p-valı & 0.033 & 0.019 & 0.031 & 0.020 & 0.020 & 0.008 & 0.015 \\
\hline Arellano-Bond (2) & -0.276 & -0.374 & -0.280 & -0.430 & -0.276 & -0.173 & -0.357 \\
\hline Arellano-Bond (2) [p-valı & 0.782 & 0.709 & 0.779 & 0.667 & 0.783 & 0.863 & 0.721 \\
\hline Observaciones & 224 & 224 & 224 & 224 & 224 & 224 & 224 \\
\hline Número de entidades & 32 & 32 & 32 & 32 & 32 & 32 & 32 \\
\hline
\end{tabular}

Nota: $* * *, * * \mathrm{y} *$ denotan significancia al 1,5 y 10 por ciento, respectivamente. Los números entre paréntesis corresponden a los errores estándar. Todas las variables explicativas se encuentran rezagadas un periodo. 1/ Se introduce en el modelo como tasa de crecimiento 2/ La variable está en proporción del PIB Manufacturero 3/En logaritmos naturales.

Fuente: Estimaciones propias.

La evidencia sugiere que la IED como proporción del PIB manufacturero (rezagada un periodo) tiene un impacto positivo y estadísticamente significativo sobre la tasa de crecimiento anual de la productividad laboral manufacturera. Dicho resultado se mantiene en todas las especificaciones. ${ }^{17}$ En específico, un incremento de un punto porcentual (pp) en la proporción de la IED manufacturera con respecto al PIB, se asocia a un aumento de 0.08

\footnotetext{
${ }^{17}$ Estos resultados van en línea con los de Alfaro (2003) que encuentra un efecto positivo de la IED en el sector manufacturero de la economía.
} 
puntos porcentuales en la tasa de crecimiento del IPLM (columna 7). Para dimensionar el efecto, podría considerarse como ejemplo la distancia promedio en el periodo 2007-2015 entre Chiapas y Chihuahua en los niveles de IED como proporción del PIB manufacturero, la cual es un poco más de 10 pp. Así, el modelo sugiere que si Chiapas lograra un incremento de 10 pp en la IED como proporción de su PIB de las manufacturas en un año dado, la tasa anual de productividad laboral en ese sector se incrementaría en promedio 0.8 pp el siguiente año.

Este resultado es consistente con otros estudios, para el caso mexicano, que presentan evidencia de una relación positiva entre la IED y la productividad laboral (Casanueva y Rodríguez 2009; Mendoza y Cabrera 2014; Olechko 2004). Una posible explicación a esto puede encontrarse en Mitze y Özyurt (2014), quienes argumentan que la IED representa una forma efectiva de crear empleo y acumular capital, lo cual, a su vez, incrementa la productividad del empleo. Otro posible canal de transmisión de la IED a la productividad laboral es a través de la llegada de empresas transnacionales que cuentan con sistemas de producción más eficientes e integrados a las cadenas de valor global en relación a su contraparte doméstica.

En referencia a las variables de control, la única que muestra un coeficiente estadísticamente significativo es la tasa de crecimiento del empleo y cuenta con el signo esperado (negativo). El resto de las variables no cuenta con significancia estadística, si bien todas de ellas cuentan con los signos esperados, excepto el de la inflación. No obstante, debe recordarse que la especificación econométrica empleada permite controlar únicamente por la posible endogeneidad de la IED y que los coeficientes reflejan relaciones de corto plazo. Así, los coeficientes correspondientes al resto de las variables deben ser tomados con precaución, pues probablemente todos ellos muestran relaciones endógenas con el crecimiento económico del sector manufacturero.

Como ejercicio adicional se estimaron las mismas especificaciones econométricas presentadas en el Cuadro 5, pero empleando MGM-AB, también conocido como estimador de diferencias del MGM. Los resultados se presentan en el Cuadro 6. En general se observa que, en todas las especificaciones la variable indicativa de la IED muestra efectos positivos y estadísticamente significativos sobre la tasa de crecimiento de la productividad laboral de 
las manufacturas. Asimismo, destaca que los coeficientes relacionados a dicha variable son mayores (prácticamente el doble) a los registrados en el Cuadro $5 .{ }^{18}$ Además, nuevamente se registra un coeficiente negativo y estadísticamente significativo de la tasa de crecimiento del empleo sobre la tasa de crecimiento de la productividad laboral. Sin embargo, el coeficiente relacionado con la infraestructura muestra un signo contraintuitivo y con significancia estadística, por lo que dicha variable podría no estar capturando toda la infraestructura de las entidades.

Cuadro 6

Efectos de la IED sobre la Tasa de Crecimiento del IPLM (MGM-AB)

\begin{tabular}{|c|c|c|c|c|c|c|c|}
\hline & $(1)$ & $(2)$ & (3) & $(4)$ & $(5)$ & (6) & $(7)$ \\
\hline Primer rezago IPML ${ }^{1 /}$ & $\begin{array}{c}0.002 \\
(0.105)\end{array}$ & $\begin{array}{c}0.006 \\
(0.110)\end{array}$ & $\begin{array}{c}0.007 \\
(0.111)\end{array}$ & $\begin{array}{c}0.005 \\
(0.108)\end{array}$ & $\begin{array}{c}0.012 \\
(0.122)\end{array}$ & $\begin{array}{c}-0.003 \\
(0.106)\end{array}$ & $\begin{array}{l}-0.007 \\
(0.118)\end{array}$ \\
\hline $\mathrm{IED}^{2 /}$ & $\begin{array}{l}0.153^{* *} \\
(0.063)\end{array}$ & $\begin{array}{c}0.153^{* *} \\
(0.070)\end{array}$ & $\begin{array}{l}0.154^{* *} \\
(0.064)\end{array}$ & $\begin{array}{l}0.152^{* *} \\
(0.069)\end{array}$ & $\begin{array}{c}0.165^{* * *} \\
(0.055)\end{array}$ & $\begin{array}{c}0.148^{* *} \\
(0.060)\end{array}$ & $\begin{array}{c}0.167^{* *} \\
(0.072)\end{array}$ \\
\hline Empleo EMIM $^{1 /}$ & $\begin{array}{c}-0.253^{* * *} \\
(0.092)\end{array}$ & $\begin{array}{c}-0.216^{* *} \\
(0.105)\end{array}$ & $\begin{array}{c}-0.249 * * * \\
(0.092)\end{array}$ & $\begin{array}{c}-0.267 * * * \\
(0.095)\end{array}$ & $\begin{array}{c}-0.234^{* * * *} \\
(0.086)\end{array}$ & $\begin{array}{c}-0.244^{* *} \\
(0.101)\end{array}$ & $\begin{array}{c}-0.237^{* *} \\
(0.108)\end{array}$ \\
\hline Infraestructura $^{3 /}$ & & $\begin{array}{c}-0.108^{* *} \\
(0.054)\end{array}$ & & & & & $\begin{array}{c}-0.105^{* *} \\
(0.051)\end{array}$ \\
\hline Escolaridad $^{3 /}$ & & & $\begin{array}{c}-0.019 \\
(0.060)\end{array}$ & & & & $\begin{array}{c}-0.036 \\
(0.069)\end{array}$ \\
\hline Exportaciones $^{3 /}$ & & & & $\begin{array}{l}-0.006 \\
(0.028)\end{array}$ & & & $\begin{array}{l}-0.014 \\
(0.023)\end{array}$ \\
\hline Inflación ${ }^{3 /}$ & & & & & $\begin{array}{c}-0.051 \\
(0.048)\end{array}$ & & $\begin{array}{c}-0.069 \\
(0.045)\end{array}$ \\
\hline Cartera $^{3 /}$ & & & & & & $\begin{array}{c}0.008 \\
(0.037)\end{array}$ & $\begin{array}{c}0.035 \\
(0.022)\end{array}$ \\
\hline Control de Endogeneidad & SI & SI & SI & SI & SI & SI & SI \\
\hline Sargan & 23.683 & 26.056 & 23.835 & 21.812 & 21.784 & 24.309 & 22.337 \\
\hline Sargan [p-value] & 0.538 & 0.405 & 0.529 & 0.647 & 0.648 & 0.502 & 0.616 \\
\hline Arellano-Bond (1) & -1.900 & -2.577 & -1.884 & -1.773 & -1.838 & -1.905 & -1.700 \\
\hline Arellano-Bond (1) [p-value & 0.058 & 0.010 & 0.060 & 0.076 & 0.066 & 0.057 & 0.089 \\
\hline Arellano-Bond (2) & -0.304 & -0.380 & -0.294 & -0.302 & -0.317 & -0.363 & -0.656 \\
\hline trellano-Bond (2) [p-value & 0.761 & 0.704 & 0.769 & 0.763 & 0.751 & 0.717 & 0.512 \\
\hline Observaciones & 192 & 192 & 192 & 192 & 192 & 192 & 192 \\
\hline Número de entidades & 32 & 32 & 32 & 32 & 32 & 32 & 32 \\
\hline
\end{tabular}

Nota: ***, ** y * denotan significancia al 1, 5 y 10 por ciento, respectivamente. Los números entre paréntesis corresponden a los errores estándar. Todas las variables explicativas se encuentran rezagadas un periodo. 1/ Se introduce en el modelo como tasa de crecimiento 2/ La variable está en proporción del PIB Manufacturero 3/En logaritmos naturales.

Fuente: Estimaciones propias.

${ }^{18}$ Baiashvili y Gattini (2020) también encuentran coeficientes mayores al emplear el estimador MGM-AB en relación al MGM-BB. 
Adicionalmente, para complementar el análisis se realizaron las estimaciones de todas las especificaciones considerando a la IED como proporción del PIB manufacturero (rezagada) como una variable exógena en lugar de endógena, los resultados se presentan en el Cuadro 7. Con excepción de la columna 7 (que se encuentra cerca del margen de significancia) en todos los casos se muestran coeficientes positivos y estadísticamente significativos de la IED como proporción del PIB en las manufacturas sobre la tasa de crecimiento del IPLM. Asimismo, es posible observar que, en la mayor parte de las especificaciones los coeficientes de la variable IED son ligeramente menores al compararlos con los del Cuadro 5. Una posible explicación a esto son las variables no observadas u omitidas en el modelo que podrían estar correlacionadas en forma negativa con la tasa de crecimiento de la IED como el desplazamiento de la inversión privada domestica (Fillat y Woerz, 2011) o el hecho de que algunas de las empresas transaccionales restrinjan la difusión de tecnologías y la innovación (Zhao y Zhang, 2010).

Otro ejercicio adicional se presenta en el Cuadro C1 del Anexo, el cual muestra las estimaciones del modelo de referencia (ecuación 1) utilizando los dos métodos empleados MGM-BB y MGM-AB pero empleando como variable dependiente el PMT analizado en la sección 3 del presente documento. En este caso se observan efectos positivos y estadísticamente significativos de la variable IED como proporción del PIB del sector manufacturas sobre la tasa de crecimiento de la productividad laboral cuando se emplea el MGM-AB. Sin embargo, el coeficiente carece de significancia estadística cuando se utiliza MGM-BB. No obstante, como se explica en la sección 3 del documento la tasa de crecimiento del IPLM representa una mejor forma de capturar la evolución de la productividad laboral que el PMT. 


\section{Cuadro 7}

Efectos de la IED sobre la Tasa de Crecimiento del IPLM (MGM-BB)

\begin{tabular}{|c|c|c|c|c|c|c|c|}
\hline & (1) & $(2)$ & (3) & (4) & (5) & (6) & $(7)$ \\
\hline Primer rezago IPML ${ }^{1 /}$ & $\begin{array}{c}0.067 \\
(0.101)\end{array}$ & $\begin{array}{l}-0.017 \\
(0.108)\end{array}$ & $\begin{array}{l}-0.019 \\
(0.125)\end{array}$ & $\begin{array}{l}-0.002 \\
(0.120)\end{array}$ & $\begin{array}{c}0.059 \\
(0.101)\end{array}$ & $\begin{array}{c}0.006 \\
(0.109)\end{array}$ & $\begin{array}{c}0.005 \\
(0.123)\end{array}$ \\
\hline $\mathrm{IED}^{2 /}$ & $\begin{array}{l}0.078 * * \\
(0.038)\end{array}$ & $\begin{array}{l}0.072 * * \\
(0.033)\end{array}$ & $\begin{array}{l}0.079 * * \\
(0.039)\end{array}$ & $\begin{array}{l}0.072^{*} \\
(0.039)\end{array}$ & $\begin{array}{c}0.072 \\
(0.044)\end{array}$ & $\begin{array}{c}0.078^{*} \\
(0.041)\end{array}$ & $\begin{array}{c}0.059 \\
(0.041)\end{array}$ \\
\hline Empleo EMIM $^{1 /}$ & $\begin{array}{l}-0.089 \\
(0.121)\end{array}$ & $\begin{array}{l}-0.226^{*} \\
(0.136)\end{array}$ & $\begin{array}{l}-0.252 \\
(0.160)\end{array}$ & $\begin{array}{l}-0.252^{*} \\
(0.137)\end{array}$ & $\begin{array}{l}-0.138 \\
(0.150)\end{array}$ & $\begin{array}{l}-0.210 \\
(0.130)\end{array}$ & $\begin{array}{l}-0.266 \\
(0.165)\end{array}$ \\
\hline Infraestructura $^{3 /}$ & & $\begin{array}{c}-0.030^{* *} \\
(0.013)\end{array}$ & & & & & $\begin{array}{l}-0.024 \\
(0.049)\end{array}$ \\
\hline Escolaridad $^{3 /}$ & & & $\begin{array}{l}0.019 * * \\
(0.008)\end{array}$ & & & & $\begin{array}{c}0.004 \\
(0.045)\end{array}$ \\
\hline Exportaciones $^{3 /}$ & & & & $\begin{array}{c}-0.014 * * * \\
(0.005)\end{array}$ & & & $\begin{array}{l}-0.023 \\
(0.031)\end{array}$ \\
\hline Inflación $^{3 /}$ & & & & & $\begin{array}{c}0.006 \\
(0.019)\end{array}$ & & $\begin{array}{c}0.004 \\
(0.021)\end{array}$ \\
\hline Cartera $^{3 /}$ & & & & & & $\begin{array}{c}-0.013^{* *} \\
(0.006)\end{array}$ & $\begin{array}{c}0.025 \\
(0.036)\end{array}$ \\
\hline Control de Endogeneidad & NO & $\mathrm{NO}$ & NO & NO & NO & NO & NO \\
\hline Sargan & 20.463 & 59.298 & 22.652 & 20.983 & 22.577 & 22.996 & 21.836 \\
\hline Sargan [p-value] & 0.429 & 0.000 & 0.306 & 0.398 & 0.310 & 0.289 & 0.350 \\
\hline Arellano-Bond (1) & -2.269 & -2.011 & -1.825 & -2.168 & -1.926 & -1.994 & -1.923 \\
\hline Arellano-Bond (1) [p-valt & 0.023 & 0.044 & 0.068 & 0.030 & 0.054 & 0.046 & 0.055 \\
\hline Arellano-Bond (2) & -0.130 & -0.614 & -0.555 & -0.188 & -0.572 & -0.396 & -0.729 \\
\hline Arellano-Bond (2) [p-valt & 0.897 & 0.539 & 0.579 & 0.851 & 0.567 & 0.692 & 0.466 \\
\hline Observaciones & 224 & 224 & 224 & 224 & 224 & 224 & 224 \\
\hline Número de entidades & 32 & 32 & 32 & 32 & 32 & 32 & 32 \\
\hline
\end{tabular}

Nota: ***, ** $\mathrm{y} *$ denotan significancia al $1,5 \mathrm{y} 10$ por ciento, respectivamente. Los números entre paréntesis corresponden a los errores estándar. Todas las variables explicativas se encuentran rezagadas un periodo. 1/ Se introduce en el modelo como tasa de crecimiento 2/ La variable está en proporción del PIB Manufacturero 3/En logaritmos naturales.

Fuente: Estimaciones propias

Como ejercicio final, en los Cuadros 8 y 9 presentan estimaciones similares a las de los Cuadro 5 y 6, pero empleando como variable dependiente la tasa de crecimiento de la productividad laboral calculada en base a una medida de valor agregado. En específico, se estima la productividad laboral utilizando la razón del Producto Interno Bruto por Entidad Federativa para el total de las industrias manufactureras a precios constantes de 2013 (publicado por INEGI), y la Población Ocupada en el sector de las manufacturas reportada por la Encuesta Nacional de Ocupación y Empleo (ENOE). Además, en lugar de emplear la 
tasa de crecimiento del empleo derivada de la EMIM (como variable explicativa) se utiliza una variable análoga construida a partir de la ENOE. ${ }^{19}$

\section{Cuadro 8}

\section{Efectos de la IED sobre la Tasa de Crecimiento del PIBMT (MGM-BB)}

\begin{tabular}{|c|c|c|c|c|c|c|c|}
\hline & (1) & $(2)$ & (3) & (4) & (5) & (6) & (7) \\
\hline Primer rezago IPML ${ }^{1 /}$ & $\begin{array}{c}-0.112 \\
(0.075)\end{array}$ & $\begin{array}{l}-0.106 \\
(0.087)\end{array}$ & $\begin{array}{l}-0.150 * \\
(0.078)\end{array}$ & $\begin{array}{l}-0.105^{*} \\
(0.055)\end{array}$ & $\begin{array}{c}-0.136 * \\
(0.072)\end{array}$ & $\begin{array}{c}-0.098 \\
(0.076)\end{array}$ & $\begin{array}{l}-0.125^{*} \\
(0.068)\end{array}$ \\
\hline IED $^{2 /}$ & $\begin{array}{c}0.068^{* *} \\
(0.028)\end{array}$ & $\begin{array}{c}0.036 \\
(0.038)\end{array}$ & $\begin{array}{c}0.040 \\
(0.045)\end{array}$ & $\begin{array}{c}0.057 * \\
(0.030)\end{array}$ & $\begin{array}{c}0.036 \\
(0.043)\end{array}$ & $\begin{array}{c}0.054 \\
(0.036)\end{array}$ & $\begin{array}{c}0.047 \\
(0.048)\end{array}$ \\
\hline Empleo $\mathrm{ENOE}_{\mathrm{t}}{ }^{1 /}$ & $\begin{array}{c}-0.695^{* * *} \\
(0.096)\end{array}$ & $\begin{array}{c}-0.726^{* * *} \\
(0.088)\end{array}$ & $\begin{array}{c}-0.746^{* * *} \\
(0.081)\end{array}$ & $\begin{array}{c}-0.689 * * * \\
(0.097)\end{array}$ & $\begin{array}{c}-0.733^{* * *} \\
(0.086)\end{array}$ & $\begin{array}{c}-0.725^{* * *} \\
(0.079)\end{array}$ & $\begin{array}{c}-0.810 * * * \\
(0.075)\end{array}$ \\
\hline Empleo $\mathrm{ENOE}_{\mathrm{t}-1}{ }^{1 /}$ & $\begin{array}{l}-0.038 \\
(0.084)\end{array}$ & $\begin{array}{l}-0.063 \\
(0.095)\end{array}$ & $\begin{array}{c}-0.109 \\
(0.090)\end{array}$ & $\begin{array}{l}-0.033 \\
(0.060)\end{array}$ & $\begin{array}{l}-0.115 \\
(0.085)\end{array}$ & $\begin{array}{l}-0.055 \\
(0.092)\end{array}$ & $\begin{array}{l}-0.100 \\
(0.084)\end{array}$ \\
\hline Infraestructura ${ }^{3 /}$ & & $\begin{array}{c}-0.021 * * \\
(0.008)\end{array}$ & & & & & $\begin{array}{l}-0.079 \\
(0.052)\end{array}$ \\
\hline Escolaridad $^{3 /}$ & & & $\begin{array}{c}0.016^{* * *} \\
(0.004)\end{array}$ & & & & $\begin{array}{c}0.052 * \\
(0.027)\end{array}$ \\
\hline Exportaciones $^{3 /}$ & & & & $\begin{array}{c}0.004 \\
(0.010)\end{array}$ & & & $\begin{array}{c}0.014 \\
(0.018)\end{array}$ \\
\hline Inflación $^{3 /}$ & & & & & $\begin{array}{c}-0.012^{* * *} \\
(0.003)\end{array}$ & & $\begin{array}{c}0.012 \\
(0.024)\end{array}$ \\
\hline Cartera $^{3 /}$ & & & & & & $\begin{array}{c}-0.010^{* * *} \\
(0.003)\end{array}$ & $\begin{array}{c}0.055^{*} \\
(0.032)\end{array}$ \\
\hline Control de Endogeneidad & SI & SI & SI & SI & SI & SI & SI \\
\hline Sargan & 28.943 & -2.345 & -2.298 & -2.795 & 30.153 & -2.553 & 22.329 \\
\hline Sargan [p-value] & 0.622 & 0.019 & 0.022 & 0.005 & 0.560 & 0.011 & 0.898 \\
\hline Arellano-Bond (1) & -2.549 & 0.196 & -0.203 & 0.341 & -2.465 & 0.396 & -2.481 \\
\hline Arellano-Bond (1) [p-value] & 0.011 & 0.845 & 0.840 & 0.733 & 0.014 & 0.692 & 0.013 \\
\hline Arellano-Bond (2) & 0.293 & 28.913 & 30.398 & 28.907 & -0.194 & 27.690 & -0.089 \\
\hline Arellano-Bond (2) [p-value] & 0.769 & 0.624 & 0.548 & 0.624 & 0.846 & 0.685 & 0.929 \\
\hline Observaciones & 224 & 224 & 224 & 224 & 224 & 224 & 224 \\
\hline Número de entidades & 32 & 32 & 32 & 32 & 32 & 32 & 32 \\
\hline
\end{tabular}

Nota: ***, ** $\mathrm{y} *$ denotan significancia al 1,5 y 10 por ciento, respectivamente. Los números entre paréntesis corresponden a los errores estándar. Todas las variables explicativas se encuentran rezagadas un periodo con excepción de Empleo ENOE t. 1/ Se introduce en el modelo como tasa de crecimiento 2/ La variable está en proporción del PIB Manufacturero 3/En logaritmos naturales.

Fuente: Estimaciones propias

\footnotetext{
${ }^{19}$ Se decidió emplear la variable contemporánea y la rezagada de la tasa de crecimiento del empleo, dado que al incluir sólo a ultima (como en las especificaciones anteriores) el signo de dicha variable era contraintuitivo y generaba resultados poco estables al cambiar de especificación. Al cambiar la variable dependiente y la medición de la tasa de crecimiento del empleo se pueden emplear diferentes especificaciones las presentadas en los Cuadros 5 y 6 . En este caso, se utilizan 3 rezagos de la variable dependiente y dos rezagos de las variables explicativas como instrumentos. Todas las especificaciones presentadas satisfacen las pruebas de Sargan y de Arellano Bond para garantizar la correcta especificación del modelo.
} 
En términos generales, el Cuadro 8 muestra que la variable de IED como proporción del PIB manufacturero, si bien cuenta con coeficientes positivos en todas las especificaciones, dichos estimadores son estadísticamente significativos en las columnas 1 y 4, y en el margen de la significancia en la columna 6, mientras que en el resto de las especificaciones la probabilidad de significancia se encuentra entre 0.3 y $0.4 .^{20}$ Los resultados son uniformes y a favor de las significancia estadística cuando se emplea el MGM-AB como método de estimación, pues en todas las especificaciones del Cuadro 9 se observa un coeficiente positivo y estadísticamente significativo de la IED (como proporción del PIB manufacturero) sobre la tasa de crecimiento del PIBMT. Asimismo, note que al igual que cuando se emplea el IPLM para medir la productividad laboral, la magnitud de los coeficientes es mayor cuando se utiliza el MGM-AB en comparación con su contraparte estimada vía el MGM-BB.

Dado que existen ciertas diferencias en los resultados (en la magnitud de los coeficientes y el grado de significancia estadística en algunas de las especificaciones) al emplear PIBMT y el IPLM es conveniente analizar las ventajas y desventajas que existen entre ellos. Como se mencionó, el IPLM al ser una medida basada en producto total es más sensible a cambios en el uso de los insumos intermedios, por lo que existe la posibilidad de que este factor sea lo que esté generando el coeficiente positivo y significativo en dichas estimaciones. Sin embargo, el IPLM hace un mejor trabajo que el PIBMT para evitar que la variación en la productividad laboral sea el resultado de un incorrecto tratamiento de los precios y/o del índice empleado para deflactar. En específico, el PIBMT se construyó empleando un índice nacional de precios al momento de deflactar, el cual no considera las variaciones la inflación de las entidades federativas. ${ }^{21}$ Estos elementos son importantes pues la evolución del PIBMT depende del índice de precios que se utiliza para deflactarlo, sobre todo si se considera que la tasa de crecimiento anual de los precios es superior a la tasa de crecimiento de la productividad laboral en el periodo analizado.

\footnotetext{
${ }^{20}$ Si se usan los errores estándar tradicionales del MGM (en lugar de los errores estándar con la corrección propuesta por Windmeijer) los coeficientes son estadísticamente significativos en todas las especificaciones.

${ }^{21}$ El Anexo A que habla sobre la construcción del IPLM describe como el INEGI da un cuidadoso tratamiento a la información relacionada a precios y del uso de ponderadores para obtener una mejor estimación por entidad federativa que la que se puede obtener por medio de un deflactor general sectorial.
} 
Cuadro 9

Efectos de la IED sobre la Tasa de Crecimiento del PIBMT (MGM-AB)

\begin{tabular}{|c|c|c|c|c|c|c|c|}
\hline & (1) & (2) & (3) & (4) & (5) & (6) & (7) \\
\hline Primer rezago IPML ${ }^{1 /}$ & $\begin{array}{c}-0.137 * \\
(0.077)\end{array}$ & $\begin{array}{c}-0.127 \\
(0.078)\end{array}$ & $\begin{array}{c}-0.166^{* *} \\
(0.068)\end{array}$ & $\begin{array}{c}-0.129 \\
(0.080)\end{array}$ & $\begin{array}{r}-0.148 \\
(0.092)\end{array}$ & $\begin{array}{l}-0.152 * \\
(0.082)\end{array}$ & $\begin{array}{c}-0.179 * * \\
(0.080)\end{array}$ \\
\hline $\mathrm{IED}^{2 /}$ & $\begin{array}{c}0.115^{* * *} \\
(0.038)\end{array}$ & $\begin{array}{c}0.121^{* * *} \\
(0.041)\end{array}$ & $\begin{array}{c}0.119 * * * \\
(0.037)\end{array}$ & $\begin{array}{c}0.140^{* *} \\
(0.058)\end{array}$ & $\begin{array}{c}0.110^{* * *} \\
(0.043)\end{array}$ & $\begin{array}{c}0.093^{* *} \\
(0.042)\end{array}$ & $\begin{array}{l}0.122^{* *} \\
(0.062)\end{array}$ \\
\hline Empleo ENOE ${ }_{t}^{1 /}$ & $\begin{array}{c}-0.773^{* * *} \\
(0.080)\end{array}$ & $\begin{array}{c}-0.770^{* * *} \\
(0.084)\end{array}$ & $\begin{array}{c}-0.789^{* * *} \\
(0.085)\end{array}$ & $\begin{array}{c}-0.785^{* * *} \\
(0.079)\end{array}$ & $\begin{array}{c}-0.767 * * * \\
(0.080)\end{array}$ & $\begin{array}{c}-0.789 * * * \\
(0.069)\end{array}$ & $\begin{array}{c}-0.799 * * * \\
(0.078)\end{array}$ \\
\hline Empleo ENOE $_{\mathrm{t}-1} 1 /$ & $\begin{array}{c}-0.071 \\
(0.059)\end{array}$ & $\begin{array}{r}-0.050 \\
(0.060)\end{array}$ & $\begin{array}{l}-0.081 \\
(0.059)\end{array}$ & $\begin{array}{l}-0.038 \\
(0.076)\end{array}$ & $\begin{array}{c}-0.089 \\
(0.076)\end{array}$ & $\begin{array}{l}-0.094 \\
(0.076)\end{array}$ & $\begin{array}{l}-0.077 \\
(0.082)\end{array}$ \\
\hline Infraestructura $^{3 /}$ & & $\begin{array}{c}-0.080^{*} \\
(0.048)\end{array}$ & & & & & $\begin{array}{c}-0.133^{* *} \\
(0.062)\end{array}$ \\
\hline Escolaridad $^{3 /}$ & & & $\begin{array}{l}0.062 * \\
(0.036)\end{array}$ & & & & $\begin{array}{c}0.045 \\
(0.043)\end{array}$ \\
\hline Exportaciones $^{3 /}$ & & & & $\begin{array}{c}-0.071^{*} \\
(0.038)\end{array}$ & & & $\begin{array}{l}-0.068 \\
(0.049)\end{array}$ \\
\hline Inflación $^{3 /}$ & & & & & $\begin{array}{l}-0.015 \\
(0.027)\end{array}$ & & $\begin{array}{l}-0.023 \\
(0.030)\end{array}$ \\
\hline Cartera $^{3 /}$ & & & & & & $\begin{array}{c}0.041 \\
(0.043)\end{array}$ & $\begin{array}{c}0.057 \\
(0.063)\end{array}$ \\
\hline Control de Endogeneidad & SI & SI & SI & SI & SI & SI & SI \\
\hline Sargan & 21.454 & 22.124 & 23.048 & 22.760 & 21.465 & 21.580 & 23.729 \\
\hline Sargan [p-value] & 0.312 & 0.278 & 0.235 & 0.248 & 0.312 & 0.306 & 0.207 \\
\hline Arellano-Bond (1) & -2.637 & -2.631 & -2.539 & -2.722 & 2.495 & -2.306 & -2.140 \\
\hline Arellano-Bond (1) [p-value] & 0.008 & 0.009 & 0.011 & 0.007 & 0.013 & 0.021 & 0.032 \\
\hline Arellano-Bond (2) & 0.261 & 0.342 & 0.032 & 0.354 & 0.012 & -0.162 & 0.619 \\
\hline Arellano-Bond (2) [p-value] & 0.794 & 0.732 & 0.974 & 0.723 & 0.990 & 0.872 & 0.536 \\
\hline Observaciones & 192 & 192 & 192 & 192 & 192 & 192 & 192 \\
\hline Número de entidades & 32 & 32 & 32 & 32 & 32 & 32 & 32 \\
\hline
\end{tabular}

Nota: ***, ** $\mathrm{y} *$ denotan significancia al 1,5 y 10 por ciento, respectivamente. Los números entre paréntesis corresponden a los errores estándar. Todas las variables explicativas se encuentran rezagadas un periodo con excepción de Empleo ENOE $_{\text {t. }} 1$ / Se introduce en el modelo como tasa de crecimiento 2/ La variable está en proporción del PIB Manufacturero 3/En logaritmos naturales.

Fuente: Estimaciones propias

Otra de las ventajas de utilizar el IPLM y no el PIBMT es que el primero realiza una contabilidad más precisa del factor trabajo. Mientras la medición de los trabajadores y las horas trabajadas en el IPLM corresponden exactamente a los mismos datos de producción (ambos generados por la EMIM), los del PIBMT son estimados a partir de la ENOE y no corresponden exactamente a las unidades de valor agregado reportadas. Además, de acuerdo 
la OECD (2001) si se usa información de las encuestas aplicadas a hogares para medir el factor trabajo debería aplicarse una corrección por migración de trabajadores entre estados, o porque los trabajadores viven en entidades distintas a donde laboran. Finalmente, la construcción del PIBMT se basa en una simple suma para contabilizar el factor trabajo mientras el IPLM hace un análisis más cuidadoso que involucra la estimación de un índice que considera promedios mensuales de ocupación y sumas ponderadas. ${ }^{22}$

Por lo tanto, los factores mencionados podrían estar influyendo en las diferencias observadas en la magnitud de los coeficientes y el grado de significancia estadística de la IED como proporción del PIB manufacturero sobre las dos medidas de productividad laboral empleadas. Así, considerando la discusión anterior, y los resultados presentados en los Cuadros 5 al 9, se sugiere que existe un efecto positivo y estadísticamente significativo de la IED (como proporción del PIB manufacturero) sobre la tasa de crecimiento de la productividad laboral en México durante el periodo de análisis. Sin embargo, este resultado no es estadísticamente significativo en algunas de las especificaciones en donde se utiliza el PIBMT para medir la productividad laboral, por lo que no se puede descartar que los resultados derivados al utilizar el IPLM sean el resultado de un uso distinto en los insumos intermedios y no de una mayor productividad laboral.

Cabe señalar que las estimaciones aquí presentadas consideran posibles problemas de endogeneidad y/o variable omitida entre la productividad laboral y la IED. No obstante, este problema puede aún persistir en otras variables incluidas en el análisis por lo que los resultados deben tomarse con precaución. Además, las estimaciones en este documento no consideran la dimensión geográfica espacial entre entidades federativas que puede ser importante en variables como la infraestructura y la IED, por lo que su estudio es importante en futuras investigaciones relacionadas con el tema. ${ }^{23}$ Adicionalmente, existen otras posibles

\footnotetext{
${ }^{22}$ Ver Anexo A y manual del INEGI sobre su construcción. De acuerdo a la OECD (2001), el uso de índices del factor trabajo constituye una mejor forma de contabilizar el factor trabajo que una simple suma de horas o trabajadores.

${ }^{23}$ La IED en una entidad federativa puede verse influenciada por la inversión extranjera directa de los estados vecinos. Por ejemplo, es más probable que se abra una planta de autopartes en una entidad vecina en donde existe una armadora automotriz, o que las inversiones se realicen en áreas geográficas con mejor infraestructura en sus alrededores.
} 
determinantes de la productividad laboral que pueden ser considerados en estudios futuros como la criminalidad y la inversión en tecnología y comunicaciones, por mencionar algunos.

\section{Conclusiones}

La productividad laboral es, en el largo plazo, el principal determinante del nivel de vida de un país, por el impacto que esta tiene sobre los salarios, los precios de los productos y los costos de las empresas. Asimismo, la productividad laboral está directamente relacionada con el crecimiento económico, el cual influye sobre los niveles de bienestar de los habitantes de un país o región. No existe un consenso en la literatura sobre los efectos de la IED en la productividad laboral, y la literatura relacionada en el tema es escasa en México.

El presente estudio utiliza la heterogeneidad en la productividad laboral manufacturera entre las entidades federativas del país con la finalidad de identificar el impacto de la IED sobre la productividad laboral manufacturera. Empleando el método generalizado de momentos, y controlando por diversas variables que influyen sobre la productividad, se muestra evidencia que indica que la IED es un determinante importante de la tasa de crecimiento de la productividad laboral manufacturera medida a través del IPLM, construido y publicado por INEGI a partir de una sola fuente (EMIM). Dichos resultados se mantienen empleando distintas especificaciones econométricas y métodos de estimación. Por su parte, cuando se mide la productividad laboral a través del PIB manufacturero por trabajador, se observan coeficientes positivos y estadísticamente significativos en todas las especificaciones estimadas por medio del MGM-AB, mientras que cuando la estimación se realiza por medio del MGM-BB, el coeficiente no resulta estadísticamente significativo en algunas especificaciones, si bien se observa un coeficiente positivo en todas ellas, y las especificaciones no significativas se encuentran, en su mayoría, no muy alejadas de los límites estadísticos de significancia.

En suma, la evidencia presentada en este documento sugiere que uno de los canales a través de los cuales la IED puede contribuir al desarrollo económico de las entidades federativas es el de aumentos en la productividad laboral, la cual a su vez influye sobre los salarios de los trabajadores y la eficiencia productiva de las empresas. Sin embargo, es necesario seguir investigando los efectos de la IED sobre la productividad laboral y el crecimiento económico en México, en investigaciones futuras podrían considerarse efectos de complementariedad 
entre la IED y la educación (Borenztein et al., 1998), o la IED y el desarrollo financiero (Alfaro et al., 2004), o la IED y el nivel de ingresos o la fortaleza de las instituciones (Baiashvili y Gattini, 2020). 


\section{Referencias}

Alfaro, L. (2003). “Foreign Direct Investment and Growth: Does the Sector Matter?” Harvard Business School.

Alfaro, L., Chanda, A., Kalemli-Ozcan, S., y Sayek, S. (2004). "FDI and Economic Growth: the Role of Local Financial Markets.” Journal of International Economics, Vol. 64, Issue 1, 89-112.

Amiti, M., y Konings, J. (2007). “Trade Liberalization, Intermediate Inputs, and Productivity: Evidence from Indonesia.” American Economic Review. Vol. 97, No.5, 161138.

Arellano, M. y Bond, S. (1991). “Some Tests of Specification for Panel Data: Monte Carlo Evidence and an Application to Employment Equations”. The Review of Economic Studies. Vol. 58, No. 2, 277-297.

Baiashvili, T., y Gattini, L. (2020). “Impact of FDI on Economic Growth: The Role of Country Income Levels and Institutional Strength.” European Investment Bank. Working Paper 2020/02.

Baltagi, B. H. y Rich, D. P. (2005). "Skill-biased Technical Change in US Manufacturing: a General Index Approach”. Journal of Econometrics, Vol. 126, No. 2, 549-570.

Basu, S., y Fernald, J. G. (1995). “Are Apparent Productive Spillovers a Figment of Specification Error?” Journal of Monetary Economics, Vol.36, 165-188.

Belorgey, N., Lecat, R., y Maury, T.-P. (2006). Determinants of Productivity per Employee: An Empirical Estimation Using Panel Data. Economic Letters, Vol. 91, 153-157.

Blundell, R., y S. Bond (1998). "Initial Conditions and Moment Restrictions in Dynamic Panel Data Models. Journal of Econometrics Vol.87, 115-143.

Borensztein, E., Gregorio, J. y Lee, J.W. (1998). "How Does Foreign Direct Investment Affect Economic Growth?” Journal of International Economics, Vol.45, No. 1, 115-135.

Bourlès, R., y Cette, G. (2005). “A Comparison of Structural Productivity Levels in Major Industrialized Countries. Documento de Trabajo No. 133. Banque de France. 
Bourlès, R., y Cette, G. (2007). "Trends in Structural Productivity Levels in the Major Industrialized Countries.” Economic Letters, Vol.95, 151-156.

Carkovic, M., y Levine, R. (2005). “Does Foreign Direct Investment Accelerate Economic Growth?” En M. Carkovic, and R. Levine, Does Foreign Direct Investment Promote Development 195.

Carlino, G.A. y R. Voith (1992). "Accounting for Differences in Aggregate State Productivity.” Regional Science and Urban Economics, Vol. 22, 597-617.

Casanueva R. C. y Rodríguez, C. A. (2009). "La Productividad en la Industria Manufacturera Mexicana: Calidad del Trabajo y Capital Humano.” Comercio Exterior, Vol.59, No.1, 16-33.

Choudhry, M. T., Marelli, E., y Signorelli, M. (2016). “Age Dependency and Labour Productivity Divergence.” Applied Economics, Vol.48, No.50, 4823-4845.

Cobbold, T. (2003). "A Comparison of Gross Output and Value-Added Methods of Productivity Estimation.” Productivity Commission Research Memorandum.

Decker, C. S., Thompson, E. C., y Wohar, M. E. (2009). "Determinants of State Labor Productivity: The Changing Role of Density.” The Journal of Regional Analysis \& Policy, $1-10$.

Dimelis, S. P., y Papaioannou, S. K. (2010). "FDI and ICT Effects on Productivity Growth: A Comparative Analysis.” European Journal of Development Research, 79-96.

Feenstra, R., Markusen, J.R. y Zeile, W. (1992). “Accounting for Growth with New Inputs: Theory and Evidence.” American Economic Review, Vol. 82, No.2, 415-421.

Fillat, C., y Woerz, J. (2011). “Good or bad? The Influence of FDI on Productivity.” The Journal of International Trade \& Economic Development, 293-328.

Gandhi, A., Navarro, S., y Rivers, D. A. (2020). "On the Identification of Gross Output Production Functions.” Journal of Political Economy Vol.128, No.8, 2973-2016.

Gullickson, W. (1995). "Measurement of Productivity Growth in U.S. Manufacturing." Monthly Labor Review, Vol.13, No.28. 
Gust, C., y Marquez, J. (2004). "International Comparisons of Productivity Growth: the Role of Information Technology and Regulatory Practices.” Labour Economics, Vol.11, 33-58.

Harris, R. (2002). "Determinants of Productivity Growth: Issues and Prospects.” En Productivity Issues in Canada, Ed. S. Rao and A. Sharpe. Calgary. University of Calgary Press.

Hejazi, W. y Safarian, A. (1999). “Trade, Foreign Direct Investment and R\&D Spillovers.” Journal of International Business Studies, Vol. 30, No.3: 491-511.

Heshmati, A. (2011). "Development and Sources of Labor Productivity in Chinese Provinces.” IZA Discussion Paper No.6263.

(INEGI) Instituto Nacional de Estadística y Geografía (2015). “Cálculo de los Î́ndices de Productividad Laboral y del Costo Unitario de la Mano de Obra 2015.” Instituto Nacional de Estadística y Geografía. 2ª Edición. México.

Jolliffe, I. T. (2002). Principal Component Analysis and Factor Analysis. Principal Component Analysis, 150-166.

Kasahara, H., y Rodrigue J. (2008). "Does the Use of Imported Intermediates Increase Productivity? Plant-Level Evidence.” Journal of Development Economy, Vol. 87, No.1,10618.

Kiviet, J. F. (1995). “On Bias, Inconsistency, and Efficiency of Various Estimators in Dynamic Panel Data Models. Journal of Econometrics, Vol. 68, 53-78.

Levinsohn, J., y Petrin, A. (2003). “Estimation Production Functions Using Inputs to Control for Unobservables.” Review of Economic Studies, Vo.70, No.2, 317-42.

Jorgenson, D.W., y Gollop, F.M. and Fraumeni, B.M. (1987). "Productivity and U.S. Economic Growth.” Harvard University Press, Cambridge.

Markusen,J. y A. Venables (1999). "Foreign Direct Investment as a Catalyst for Industrial Development.” European Economic Review Vo.43, 335-56.

Mei, H. y Ben-Lon Chen. (2000), "Labour Productivity of Small and Manufacturing Firms: the case of Taiwan”, Contemporary Economic Policy, Vol.18, No.3, 270-283. 
Mendoza Cota, J. E. y Cabrera Pereyra J. A. (2014). “Trabajo Calificado, Especialización y

Productividad laboral Urbana en la Frontera Norte de México: un Análisis de Panel de Efectos Mixtos.” Investigación Económica, Vol. LXXIII, No. 287, 89-119.

Mitra, A., Varoudakis, A., y Veganzones-Varoudakis, M. A. (2002). "Productivity and Technical Efficiency in Indian states’ Manufacturing: the Role of Infrastructure.” Economic Development and Cultural Change, Vo.50, No.2, 395-426.

Mitze, T. y Özyurt, S. (2014). “The Spatial Dimension of Trade-and FDI-driven Productivity Growth in Chinese Provinces: A Global Cointegration Approach”. Growth and Change, Vol. 45, No. 2, 263 - 291.

Olechko, D. F. (2004). “Inversión Extranjera y Productividad en México.” Investigación Económica, 147-173.

(OECD) Organisation for Economic Co-operation and Development (2001). "Measuring Productivity.” OECD Manual.

(OIT) Organización Internacional del Trabajo (2008). “Informe V. Calificaciones para la Mejora de la Productividad, el Crecimiento del Empleo y el Desarrollo.” Conferencia Internacional del Trabajo 97a Reunión. Disponible en: http://www.ilo.org/

Oulton N. y O’Mahony, M. (1994). “Productivity and Growth, Cambridge University Press, Cambridge.

Ram, R. y K. Zhang (2002). "Foreign Direct Investment and Economic Growth: Evidence from Cross-Country Data for the 1990s,” Economic Development and Cultural Change, Vol.51, 205-15.

Romer, Paul M. (1990), “Endogenous Technical Change”, Journal of Political Economy, Vol. 98. No.5, S71-S102.

Solow, R.M. (1957), “Technical Change and the Aggregate Production Function.” The Review of Economics and Statistics, Vol.39, No.3, 312-320. 
Vergeer, R. y Kleinknecht, A. (2002). “Do Labour Market Reforms Reduce Labour Productivity Growth? A panel data analysis of 20 OECD countries (1960-2004).” International Labour Review, Vol.153, Issue.3, 365-393.

Windmeijer, F. (2005). “A Fnite Sample Correction for the Variance of Linear Efficient twostep GMM Estimators.” Journal of Econometrics, Vol. 126, 25-51.

Yazdan, G. F, y Hossein, S. M. (2013). “FDI and ICT Effects on Productivity Growth.” Procedia-Social and Behavioral Sciences, Vol.93, 1710-1715.

Zhao, Z. y Zhang, K. H. (2010). “FDI and Industrial Productivity in China: Evidence from Panel Data in 2001-06.” Review of Development Economics, Vol.14, No.3, 656-665. 


\section{Anexo A: Cálculo de los Índices de Volumen Físico de la Producción Total y el Índice de Horas Trabajadas. ${ }^{24}$}

El IPLM estimado por INEGI, se define como la razón entre el índice de volumen físico de la producción total y el índice de horas trabajadas en el sector manufacturero. Ambos índices elaborados primordialmente con información de la Encuesta Mensual de la Industria Manufacturera (EMIM).

Es de suma relevancia mencionar que el Índice de volumen físico de la producción total conlleva un cálculo complejo que enseguida se detalla de manera descriptiva:

i. Se obtiene los precios para cada producto en el año base y en los demás periodos, dividendo el promedio mensual del valor de la producción del producto i entre el promedio mensual del volumen de la producción del producto i.

ii. Con los precios promedio se obtiene el valor mensual de la producción a precios constantes del año base para la clase c, por medio de la sumatoria del producto del precio medio del producto i y el volumen de la producción medio mensual del producto i en el año base, divido entre 12 .

iii. Con estos mismos precios promedio se obtiene el valor de la producción a precios constantes de los productos en volumen y valor de la clase c en el mes n por medio de la sumatoria del producto de los precios medios del producto i en el año base y el volumen de la producción del producto i en el mes n.

iv. Posteriormente, al dividir el valor de la producción de los productos medidos en volumen y valor en la clase c a precios corrientes en el mes n entre el valor de la producción promedio mensual de los productos medidos en volumen y valor en la clase c en el año base 2008, se estima el Índice de valor de la producción de los productos medidos en volumen y valor en la clase c a precios corrientes en el mes n.

v. El índice de precios implícitos del conjunto de los productos medidos en volumen y valor se obtiene al dividir el índice de valor y el índice de volumen, obtenidos previamente.

\footnotetext{
${ }^{24}$ El procedimiento descrito es un resumen metodológico. El procedimiento detallado se puede encontrar en INEGI (2015).
} 
vi. Del grupo de productos medidos únicamente en valor, se agrupan tres conceptos: a) productos secundarios dentro de la clase de actividad; b) desechos y subproductos; c) otros productos no genéricos.

Se deflactan por medio de la construcción del índice de precios implícitos, los cuales están definidos como la razón de Índice de valor de la producción de los productos medidos en volumen y valor de la clase c en el mes n, y el índice de volumen de los productos medidos en volumen y valor de la clase c en el mes $n$.

vii. El mencionado índice de precios implícitos para la clase c se utiliza para deflactar el conjunto de productos para los que solo se tiene el valor de la producción de la misma clase.

viii. Las actividades adicionales al giro principal de las empresas manufactureras, también se registran en la EMIM. Una de ellas es el concepto denominado ingresos por maquila, submaquila y remanufactura, el cual resulta de los montos percibidos por los establecimientos manufactureros por concepto de servicios de transformación de materias primas o bienes intermedios propiedad de terceros. Para el cálculo de esta información se consulta la Encuesta Industrial Anual (EIA) y se da un tratamiento estadístico especial por medio de factores de ajuste, construyendo el valor estimado de la producción bruta maquilada al mercado extranjero de la clase c en el mes n tanto a precios corrientes como a precios constantes.

ix. Por otro lado, con la información de la propia EMIM, se estima el valor de los ingresos por maquila, submaquila y remanufactura provenientes del mercado nacional a precios constantes en la clase c en el mes n.

X. Al contar con los valores necesarios a precios constantes (Promedio mensual del valor de la producción de los productos medidos en volumen y valor de la clase c en el año base 2008 (y en el mes n), Promedio mensual del valor de la producción de los productos medidos solo en valor de la clase c en el año base 2008 (y en el mes n), Promedio mensual del valor estimado de la producción bruta maquilada al mercado extranjero de la clase c en el año base 2008 (y en el mes n), Promedio mensual de los ingresos por maquila, submaquila y remanufactura provenientes del mercado nacional de la clase c en el año base 2008 (y en el mes n)), se hace la agregación de los cuatro componentes de la producción manufacturera tanto para el año base como para cada uno de los meses y años, obteniendo el valor total 
promedio mensual de la producción de la clase c en el año base 2008, así como el valor total promedio mensual de la producción a precios constantes de la clase c en el mes n.

xi. Con ambos valores se estima el Índice de volumen físico de la producción total al dividir el valor total promedio mensual de la producción a precios constantes de la clase c en el mes n entre el valor total promedio mensual de la producción de la clase c en el año base 2008.

xii. Este procedimiento se utiliza para estimar los índices de volumen físico de la producción total para las 240 clases de actividad.

xiii. Posteriormente se construyen los índices ponderados de las 86 ramas. Los ponderadores corresponden a la participación del valor de la producción de cada clase en el valor de la producción total en el año base (siendo la suma de los ponderadores de clase de una rama igual a uno).

xiv. De manera análoga, el índice de volumen de la producción de una rama se estima por medio de la sumatoria de los productos de los índices de volumen físico de la producción de cada clase por su ponderador correspondiente.

XV. El mismo procedimiento se aplica para obtener los índices de volumen físico de la producción de los 21 subsectores y finalmente el índice de volumen físico del sector de las manufacturas.

Por otro lado, el Índice de personal ocupado total para la clase c en el mes n se calcula por medio de la razón entre el personal ocupado total en la clase c en el mes n y el promedio mensual del personal ocupado total en la clase c en el año base 2008. Para calcular el índice de personal ocupado de los niveles, rama, subsector y sector se utilizan sumas ponderadas. Mientras el Índice de horas trabajadas en la clase c en el mes n, se calcula dividiendo las horas trabajadas en la clase c en el mes n entre el promedio mensual de las horas trabajadas en la clase c del año base 2008. Análogamente a lo realizado con el índice de personal ocupado para los diversos niveles, se realiza una suma ponderada para obtener el índice de horas trabajadas a nivel rama, subsector y sector (para mayores detalles consultar manual del INEGI sobre su construcción). 


\section{Anexo B: Construcción del Índice de Componentes Principales}

Para medir el nivel de infraestructura por entidad en este trabajo se utilizó un índice construido por medio del Análisis de Componentes Principales. El objetivo de este procedimiento es describir la variación de un conjunto de datos por medio de la transformación de estos en un nuevo conjunto de datos con mayor parsimonia. Para lograr esto, se construye un grupo de variables no correlacionadas, donde cada una de estas es una combinación lineal de los datos originales. Es decir, el análisis de componentes principales realiza una transformación de las variables $y_{1 i} \ldots y_{p i}$, en otro conjunto de variables $z_{1 i} \ldots z_{p i}$, con $q<p$. Entonces tenemos,

$$
\begin{aligned}
& z_{1 i}=a_{11} y_{1 i}+a_{12} y_{2 i}+\cdots+a_{1 p} y_{p i} \\
& z_{2 i}=a_{21} y_{1 i}+a_{22} y_{2 i}+\cdots+a_{2 p} y_{p i} \\
& \begin{array}{l}
\cdot=\cdot \\
\cdot=\cdot+\ldots+ \\
\cdot=\cdot+\ldots+
\end{array} \\
& z_{q i}=a_{q 1} y_{1 i}+a_{q 2} y_{2 i}+\cdots+a_{q p} y_{p i}
\end{aligned}
$$

Se deriva a la variable $z_{1 i}$ que explica la mayor parte de la varianza de los datos originales, en seguida se busca una variable $z_{2 i}$ que no esté correlacionada con $z_{1 i}$ y sea la segunda en importancia en términos de contabilizar la varianza de la información, y así se continua el procedimiento hasta obtener la variable $z_{q i}$, que es la que explica la menor parte de la varianza. Los coeficientes $a_{11}, \ldots, a_{1 p}$ que ponderan a las variables originales en la construcción de los componentes principales, están construidos de manera que la varianza muestral de cada uno sea máxima. La suma de los cuadrados de estos coeficientes está restringida a 1. Adicionalmente, la correlación muestral entre los coeficientes de las variables originales $y_{1 i}, \ldots, y_{p i}$ debe ser igual a cero. Para mayores detalles técnicos se puede consultar la obra de Jolliffe (2002).

Para este trabajo se construyó una base de datos con información promedio de nueve variables que representan distintos tipos de infraestructura para el periodo sobre el que se realizaron las estimaciones. 


\section{Cuadro B1}

Variables incluidas en la construcción del Índice de Componentes Principales

\begin{tabular}{|c|c|c|}
\hline Variable & Descripción & Fuente \\
\hline Longitud de carreteras $^{1 /}$ & Kilómetros de carreteras pavimentadas & INEGI \\
\hline Longitud de vías ferreas $^{1 /}$ & Kilómetros de vías ferreas & INEGI \\
\hline Gas oline ras $^{2 /}$ & Gasolineras establecidas promedio & INEGI \\
\hline Parque vehicular ${ }^{2 /}$ & Número total de vehículos en circulación & INEGI \\
\hline Densidad telefónica & Número de suscripciones telefónicas por cada cien habitantes & INEGI \\
\hline Electricidad & Consumo de energía eléctrica (Gigawatts-hora) & CFE \\
\hline Caje ros $^{2 /}$ & Número total de cajeros & INEGI \\
\hline Cuartos totales & Cuartos totales para hospedaje & INEGI \\
\hline Bibliotecas $^{2 /}$ & Bibliotecas en operación & INEGI \\
\hline
\end{tabular}

$1 /$ variables estandarizadas con la superficie del país

2/ variables estandarizadas con la población.

Fuente: Elaboración propia.

En el cuadro B2 se observan los componentes principales para las siete variables de interés. En la segunda columna se muestra el valor propio de cada componente, es decir, la varianza asociada a cada uno, la tercera columna corresponde a la proporción de la varianza explicada, mientras la cuarta columna ilustra los valores correspondientes a la $R^{2}$ acumulada. El criterio para elegir el número de componentes principales que se utilizaron para construir el índice de infraestructura, consistió en tomar el número de componentes necesarios hasta lograr una $R^{2}$ acumulada al menos de .90. Bajo este precepto, en el cálculo del índice de infraestructura utilizado en nuestras estimaciones, se tomaron en cuenta los primeros seis componentes del análisis realizado, los cuales explican el 93 por ciento de la varianza total de los datos.

Cuadro B2

Análisis de Componentes Principales

\begin{tabular}{lccc}
\hline Componentes & Valores propios & Proporción & R2 acumulada \\
\hline Componente 1 & 3.14 & 0.35 & 0.35 \\
Componente 2 & 1.94 & 0.22 & 0.56 \\
Componente 3 & 1.04 & 0.12 & 0.68 \\
Componente 4 & 0.98 & 0.11 & 0.79 \\
Componente 5 & 0.70 & 0.08 & 0.87 \\
Componente 6 & 0.52 & 0.06 & 0.93 \\
Componente 7 & 0.32 & 0.04 & 0.96 \\
Componente 8 & 0.23 & 0.03 & 0.99 \\
Componente 9 & 0.12 & 0.01 & 1.00 \\
\hline
\end{tabular}

Fuente: Elaboración propia con datos de INEGI y CFE. 
Con los seis componentes seleccionados se formó el índice de infraestructura a través de una combinación lineal de estos, empleando como ponderador la proporción de la varianza normalizada que explica cada uno de ellos con respecto a la varianza total. Finalmente, dato que este índice contenía valores positivos y negativos se aplicó un proceso de normalización para que tomara rangos entre cero y uno.

El cuadro B3 contiene información acerca de los estadísticos descriptivos de las variables utilizadas para construir el índice de componentes principales. Es posible observar que todas las variables excepto la densidad telefónica registraron crecimiento entre el primer y último año del periodo de análisis. La longitud de vías férreas fue la variable de infraestructura que presentó un crecimiento más modesto, seguida del número de bibliotecas públicas en operación y el número de cuartos totales, mientras la longitud de carreteras creció en más de 8 por ciento y el consumo de electricidad aumentó un 17 por ciento. Por otro lado, el número de gasolineras, así como el parque vehicular total registraron variaciones positivas de 41 y 50 por ciento, y el número de cajeros fue la variable que presentó un mayor crecimiento, al incrementar en 60 por ciento entre el año 2007 y 2015. Finalmente, la densidad telefónica tuvo un decremento de 18 por ciento, lo que puede estar reflejando la sustitución de telefonía fija por telefonía celular.

\section{Cuadro B3}

Estadísticos Descriptivos de Variables de Infraestructura

\begin{tabular}{lrrrrr}
\hline & \multicolumn{2}{c}{2007} & & \multicolumn{2}{c}{2015} \\
\cline { 2 - 3 } \cline { 5 - 6 } \cline { 5 - 6 } & Promedio & $\begin{array}{c}\text { Desviación } \\
\text { Estándar }\end{array}$ & & Promedio & $\begin{array}{c}\text { Desviación } \\
\text { Estándar }\end{array}$ \\
\hline Longitud de carreteras & $11,252.3$ & $7,030.1$ & & $12,196.9$ & $7,612.8$ \\
Gangitud de vías ferreas & 833.7 & 659.1 & & 835.3 & 659.0 \\
Parque vehicular & 248.1 & 162.7 & & 350.3 & 225.9 \\
Densidad telefónica & $835,849.9$ & $735,551.0$ & & $1,256,427.0$ & $1,305,011.0$ \\
Electricidad & 16.8 & 7.0 & & 13.8 & 7.0 \\
Cajeros & $5,639.7$ & $4,259.9$ & & $6,631.3$ & $4,873.9$ \\
Cuartos totales & 866.6 & 980.3 & & $1,386.6$ & $1,371.8$ \\
Bibliotecas & $19,951.3$ & $15,783.0$ & & $20,584.7$ & $18,136.5$ \\
\hline
\end{tabular}

Nota: Las variables se presentan sin estandarizar. 


\section{Anexo C}

\section{Cuadro C1}

Efectos de la IED sobre la Tasa de Crecimiento del PMT, MGM-BB(1) y MGM-AB(2)

\begin{tabular}{|c|c|c|}
\hline & (1) & (2) \\
\hline Primer rezago IPML ${ }^{1 /}$ & $\begin{array}{l}-0.071 \\
(0.069)\end{array}$ & $\begin{array}{l}-0.112 \\
(0.092)\end{array}$ \\
\hline $\mathrm{IED}^{2 /}$ & $\begin{array}{c}0.010 \\
(0.078)\end{array}$ & $\begin{array}{l}0.129 * \\
(0.076)\end{array}$ \\
\hline Empleo EMIM $^{1 /}$ & $\begin{array}{l}-0.031 \\
(0.104)\end{array}$ & $\begin{array}{c}0.015 \\
(0.176)\end{array}$ \\
\hline Infraestructura ${ }^{3 /}$ & $\begin{array}{c}0.064 \\
(0.046)\end{array}$ & $\begin{array}{c}0.046 \\
(0.076)\end{array}$ \\
\hline Escolaridad $^{3 /}$ & $\begin{array}{l}-0.067 \\
(0.072)\end{array}$ & $\begin{array}{c}-0.224^{* * *} \\
(0.069)\end{array}$ \\
\hline Exportaciones $^{3 /}$ & $\begin{array}{c}-0.028 \\
(0.039)\end{array}$ & $\begin{array}{l}-0.079 \\
(0.088)\end{array}$ \\
\hline Inflación ${ }^{3 /}$ & $\begin{array}{c}0.041 \\
(0.052)\end{array}$ & $\begin{array}{l}0.117^{*} \\
(0.066)\end{array}$ \\
\hline Cartera $^{3 /}$ & $\begin{array}{c}0.035 \\
(0.038)\end{array}$ & $\begin{array}{c}0.027 \\
(0.038)\end{array}$ \\
\hline Control de Endogeneidad & SI & SI \\
\hline Sargan & 19.927 & 26.464 \\
\hline Sargan [p-value] & 0.993 & 0.383 \\
\hline Arellano-Bond (1) & -2.530 & -1.688 \\
\hline Arellano-Bond (1) [p-value] & 0.011 & 0.091 \\
\hline Arellano-Bond (2) & -0.640 & -0.967 \\
\hline Arellano-Bond (2) [p-value] & 0.522 & 0.333 \\
\hline Observaciones & 224 & 192 \\
\hline Número de entidades & 32 & 32 \\
\hline
\end{tabular}

Nota: ***, ** y* denotan significancia al 1, 5 y 10 por ciento, respectivamente. Los números entre paréntesis corresponden a los errores estándar. Todas las variables explicativas se encuentran rezagadas un periodo. 1/ Se introduce en el modelo como tasa de crecimiento 2/ La variable está en proporción del PIB Manufacturero 3/En logaritmos naturales.

Fuente: Estimaciones propias. 